\title{
Piezoelectric Organic Crystals Autonomously Recombine and Self- heal Fracture with Precise Order
}

Surojit Bhunia ${ }^{1,2}$, Shubham Chandel ${ }^{3}$, Sumanta Kumar Karan ${ }^{4}$, Somnath Dey ${ }^{5}$, Akash Tiwary ${ }^{3}$, Susobhan Das ${ }^{1}$, Nishkarsh kumar ${ }^{3}$, Rituparno Chowdhury ${ }^{1}$, Saikat Mondal $^{1,2}$, Amit Mondal ${ }^{1}$, Bhanu Bhusan Khatua ${ }^{4}$, Nirmalya Ghosh ${ }^{2,3 *}$, C. Malla Reddy ${ }^{1,2 *}$

1Department of Chemical Sciences, Indian Institute of Science Education and Research (IISER) Kolkata 741246, Nadia, West Bengal (India).

${ }^{2}$ Centre for Advanced Functional Materials (CAFM), Indian Institute of Science Education and Research (IISER) Kolkata 741246, Nadia, West Bengal (India).

${ }^{3}$ Department of Physical Sciences, Indian Institute of Science Education and Research (IISER) Kolkata 741246, Nadia, West Bengal (India).

${ }^{4}$ Materials Science Centre, Indian Institute of Technology Kharagpur, Kharagpur 721302, India.

Institute of Crystallography, RWTH Aachen University, Jägerstraße 17-19, 52066 Aachen, Germany

*Correspondence to: cmallareddy@gmail.com (C.M.R.); nghosh@iiserkol.ac.in (N.G.)

\begin{abstract}
Living tissue uses stress-accumulated electrical charge to close wounds, yet to-date this piezoelectric effect has not been realised in self-repairing synthetic materials which are typically soft amorphous materials requiring external stimuli, prolonged physical contact and long healing times (often $>24 \mathrm{~h}$ ). Here we overcome many of these challenges using piezoelectric organic crystals, which upon mechanical fracture, instantly recombine without any external direction, autonomously self-healing in milliseconds with remarkable crystallographic precision. Atomic-resolution structural studies reveal that a 3D hydrogen bonding network, with ability to store stress, facilitates generation of stress-induced electrical charges on the fractured crystals, creating an electrostatically-driven precise recombination of the pieces via a diffusionless instant self-healing, as supported by spatially-resolved birefringence experiments. Perfect, instant self-healing creates new opportunities for deployment of molecular crystals in robust miniaturised devices, and may also spur development of new molecular level repair mechanisms in complex biomaterials.
\end{abstract}

Main Text: Piezoelectric effect induced electric charges are known to trigger selfhealing in mechanically damaged natural biomaterials (1). In unnatural polymers, gels and composites, various strategies have been employed to mimic the nature (2-14), but approaches that directly use piezoelectricity as an inherent material property are absent. More importantly, almost all the self-healing materials are soft, amorphous and need stimuli such as heat, light, solvent or a chemical healing agent, yet universally all materials fail when the broken pieces fall apart. Another hardly accomplished but attractive goal in materials science is to couple the self-healing with crystallinity $(2,3$, $6,15,16)$. Poor diffusion in the densely packed and relatively hard ordered crystals precludes autonomous healing and makes crystallographically precise reordering extremely difficult, even under long time periods, annealing, mechanical compression or solution treatment $(15,16)$. 
Self-healing property coupled with atomically precise long range order in materials is critical for a number of key technologies. For instance, many devices that function based on precision positioning in the microelectronics technologies require precisely oriented, highly crystalline piezoelectrics. Piezoelectric materials are required to withstand prolonged mechanical loading unloading cycles in many applications; hence fracture healing ability is critical to boost up their durability (17). Despite the immense potential of highly crystalline materials in numerous applications related to optical, electrical, energy, biomedical, soft-robotics, and artificial skins, only a few reports exist on self-healing in crystalline materials with little understanding of the mechanisms (1821). Recently, it has been shown that self-healing of cracks and unusually large volume changes occur in hybrid macromolecular ferritin-hydrogel crystals, however the entire process requires modulation of spatial gradient of salt concentration in solution conditions (15). Self-healing is reported in single crystals of soft boronic esters (with an elastic modulus, $E=<2 \mathrm{MPa}$ ), utilizing dynamic covalent chemistry under moisture conditions and prolonged contact periods of $\sim 24 \mathrm{hrs}$, and more importantly, the macroscopic cracks remain visible demonstrating the challenge in retaining the integrity of crystals (16).

Here we show an alternative approach to use the inherent piezoelectric effect of organic single crystals for achieving a truly autonomous self-healing, that overcomes many of the existing disadvantages in unnatural self-healing materials. These crystals, with an order of magnitude higher stiffness and hardness as compared to most other known self-healing materials, can autonomously recombine even when the fractured pieces are physically separated, and undergo ultra-fast temporal self-healing (milliseconds) with crystallographically precise or crystal-clear ordering. Moreover, the excellent single crystalline nature of the self-healing crystals aided understanding of the molecular level mechanisms using atomic resolution structures from single crystal $\mathrm{X}$-ray diffraction technique. The degree of order in the healed crystals has been quantitatively revealed up to nano-scale spatial resolution using classical birefringence measurements. 


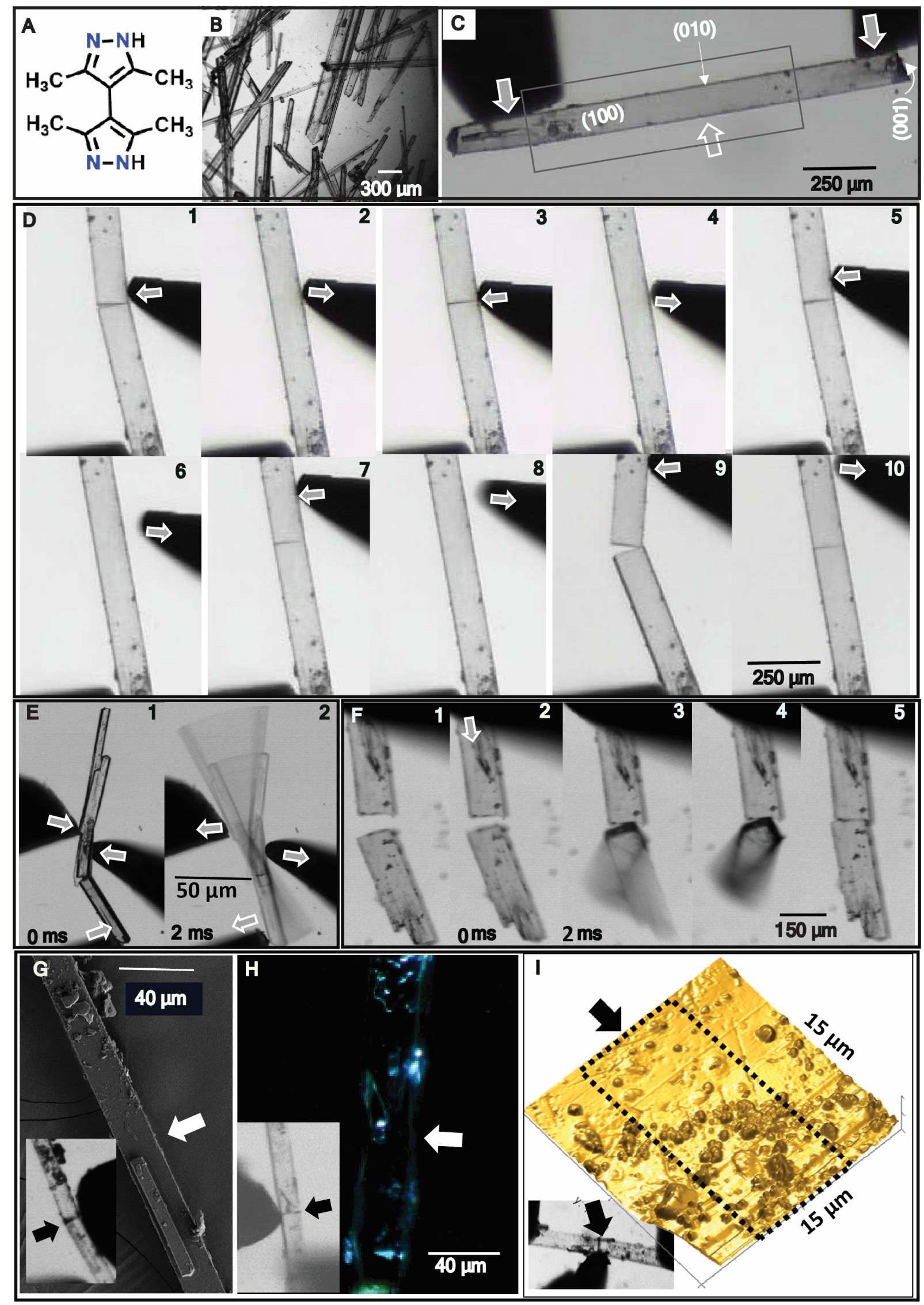

Fig. 1. Micrographs of crystals of 1 exhibiting self-healing. (A) Molecular structure. (B) Pristine single crystals. (C) A crystal used for (D) repeat self-healing cycles. (E) and $(F)$ show fast self-propelling mechanical actuation and auto-recombination of 
fractured pieces of crystals of 1. (G) Scanning electron micrograph, (H) Dark-field optical image and (I) Atomic force microscopy image of the neatly-healed crystals with no sign of cracks (insets: In $\mathrm{G}, \mathrm{H}, \mathrm{I}$ video grabs of the corresponding crystals at the cracked stage).

The compound, 3,3',5,5'-tetramethyl-4,4'-bipyrazole (Fig. 1A) is synthesized in gram scale (see supporting information, method section) and crystallized from DMSO solution at ambient conditions. The bipyrazole single crystals (hereafter, $\mathbf{1}$; length 3$5 \mathrm{~mm}$; width $=\sim 0.2-0.3 \mathrm{~mm}$ ) (Fig. 1B, 1C), when subjected to a three-point bending test, fracture via propagation of a linear crack. When the force is withdrawn, the two pieces self-propel and rejoin in the blink of an eye aided by a strong attractive force between the two broken ends. In addition, the pieces land onto each other with a remarkably perfect alignment and instantly self-heal in $<1 \mathrm{~ms}$ time scale (Fig. 1D, Fig. S1, Supporting text section 1). The crack generation and self-healing could be repeated multiple times in a crystal (Fig. 1D). Unlike all other known self-healing materials, the fractured pieces of crystal $\mathbf{1}$ can recombine even when physically separated, within a certain threshold distance. The pieces jump on to each other, travelling with a honeybee wing-like motion with acceleration of $\sim 2.2 \mathrm{~m} / \mathrm{sec}^{2}(\mathrm{Fig} .1 \mathrm{E}$, F, Fig. S2). Remarkably, the perfectly self-healed (hereafter, neatly-healed) crystals were indistinguishable from the pristine (as grown) crystals, and showed no sign of cracks, even when examined employing polarized optical microscope, scanning electron microscope (SEM) (Fig. 1G, Fig. S3, S4), dark-field imaging microscope (Fig. $1 \mathrm{H}$, Fig. S5) and atomic force microscope (AFM) techniques (Fig. 1I). We also observed instantaneous healing of millimetre length scale cracks, generated by a uniaxial compression of a crystal using external stress (Fig. S6, S10). The repeatability of the self-healing cycles depended on the way force was applied and landing direction of the two pieces (Fig. 1D10, 1E5; Fig. S4). 


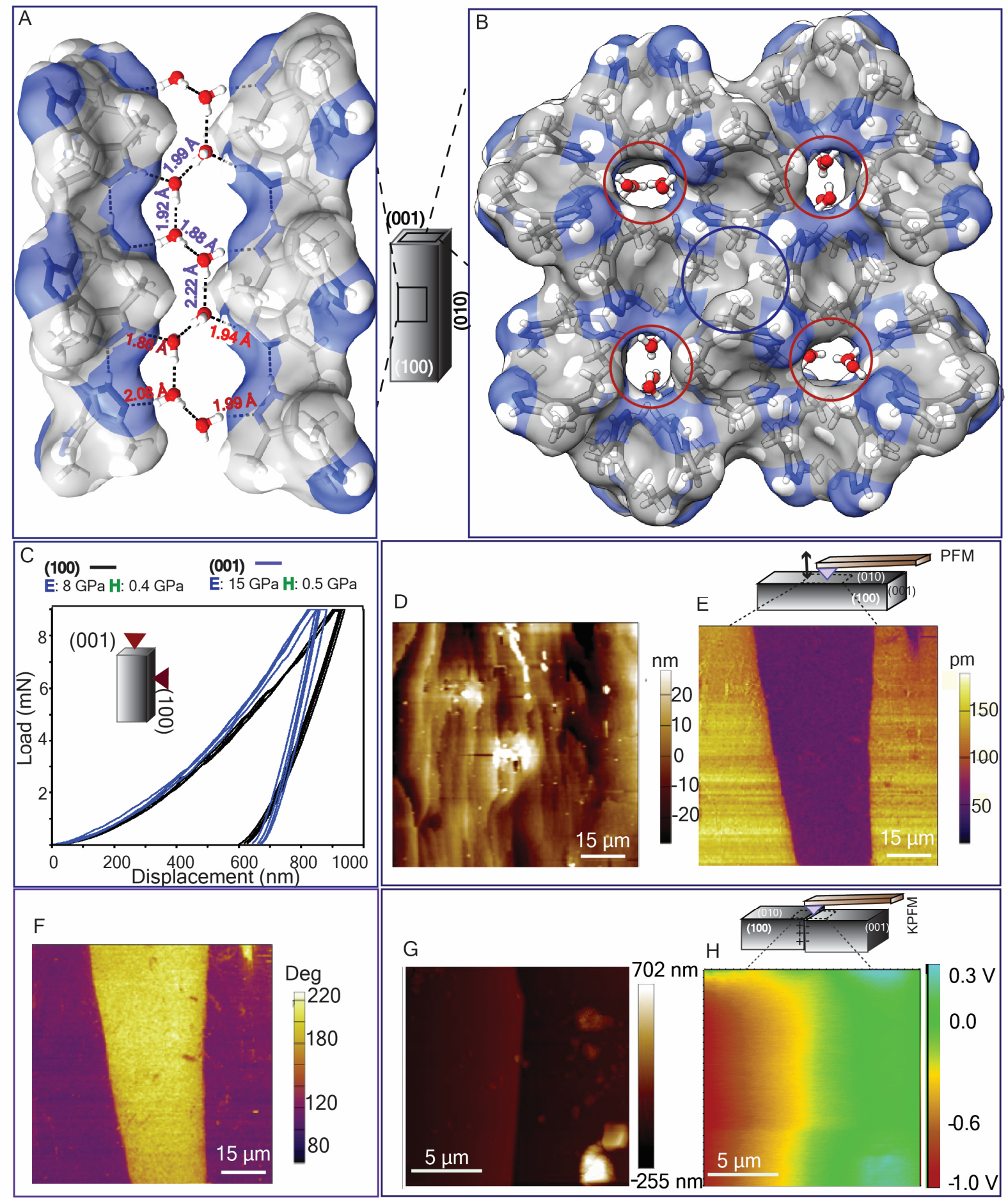

Fig. 2. Structure and physical properties of crystal 1. A) Display of 1D water chain along crystal length connecting the molecules of 1. B) Crystal packing (view II to caxis) with the hydrophilic zones of water channels (red circles) surrounded by closepacked methyl-groups (marked by blue circles). C) Load-displacement $(P-h)$ curves obtained from nanoindentation experiments on different faces of pristine crystal of 1. D) Topography, E) amplitude and F) phase images obtained from PFM on the side face of the crystal. G) Topography image and H) surface potential maps obtained from KPFM experiments on the crack-line of an imperfectly-healed crystal. 
Structure determination of pristine samples by single crystal X-ray diffraction technique revealed that the solid sample 1 is a novel monohydrate form of the bipyrazole, having tetragonal $P_{4}$ polar space group. The asymmetric unit contains four sets of independent bipyrazole and water molecules each $\left(Z^{\prime}=4\right)$ (see Fig. S7). The crystals grow along the $c$-axis with two crystallographically equivalent side faces, (100) and (010) (Fig. 2). The water molecules form an infinite one dimensional zig-zag chain via $\mathrm{O}-\mathrm{H} \cdots \mathrm{O}$ cooperative hydrogen bonds parallel to the crystal length (c-axis) (Fig. 2A, Fig. S7A). The adjacent parallel water chains are bridged in a perpendicular direction by bipyrazole molecules via $\mathrm{O}-\mathrm{H} \cdots \mathrm{N}$ and $\mathrm{N}-\mathrm{H} \cdots \mathrm{O}$ hydrogen bonding interactions (Fig. S7C). In addition, the bipyrazole molecules also interact with themselves via $\mathrm{N}-\mathrm{H} \cdots \mathrm{N}$ interactions parallel to the water chains (Fig. S7B). As a result, a strong three dimensional (3D) cooperative hydrogen bonding network is formed. The methyl groups of bipyrazoles close pack, within the 3D hydrogen bonded network (Fig. 2B) (see Table. $\mathrm{S} 1$ for $\mathrm{H}$-bonding information).

The hardness $(H$; resistance to plastic deformation) and elastic modulus ( $E$; resistance to elastic deformation) of the crystal $\mathbf{1}$ measured by nanoindentation technique suggests that this crystal is an order of magnitude harder $(H: 0.4-0.5 \mathrm{GPa})$ and stiffer (E: $8 \mathrm{GPa}$ on (100)/(010); $15 \mathrm{GPa}$ on (001)) as compared to most other self-healing materials (Table S2), but comparable to typical ordered organic crystals (Fig. 2C) (22). Piezoresponse force microscopy (PFM) on the equivalent $(100) /(010)$ faces of the polar single crystal 1 confirmed its excellent piezoelectric property and existence of distinct polarization domains (Fig. 2D-F). The magnitude of the piezoelectric response is extracted from amplitude (Fig. 2E), while the domain structure is evident from the phase image (Fig. 2F) (23). The out-of-plane piezoelectric coefficient is estimated to be $\sim 5.51 \mathrm{pm} / \mathrm{V}( \pm 0.046)$. Further, measurement by a piezometer using the microcrystalline bulk sample containing randomly oriented particles, revealed a maximum $d_{33}$ of $-13 \mathrm{pC} / \mathrm{N}$ with average values ranging $~ 8.5-9.5 \mathrm{pC} / \mathrm{N}$ (Fig. S8, supporting text section 2) Unlike single crystal samples, the microcrystalline palletsamples have contributions from many crystal facets due to their random orientation with respect to the direction of applied external stress.

Furthermore, we mapped the nanoscale local electrostatic surface potential of different crystals using Kelvin probe force microscopy (KPFM) (24). A freshly created (001)/(001) face by fracturing perpendicular to the needle axis, showed a large surface potential of $4.7 \mathrm{~V}$ (Fig. S9). This can be attributed to the fact that piezoelectric materials generate electrical charges under mechanical stress $(1,25-26)$. The experiments on (100)/(010) faces of an imperfectly-healed crystal also showed a small, but noticeable residual opposite potential $(\sim 0.2 \vee$ to $-0.6 \quad V)$ on either side 


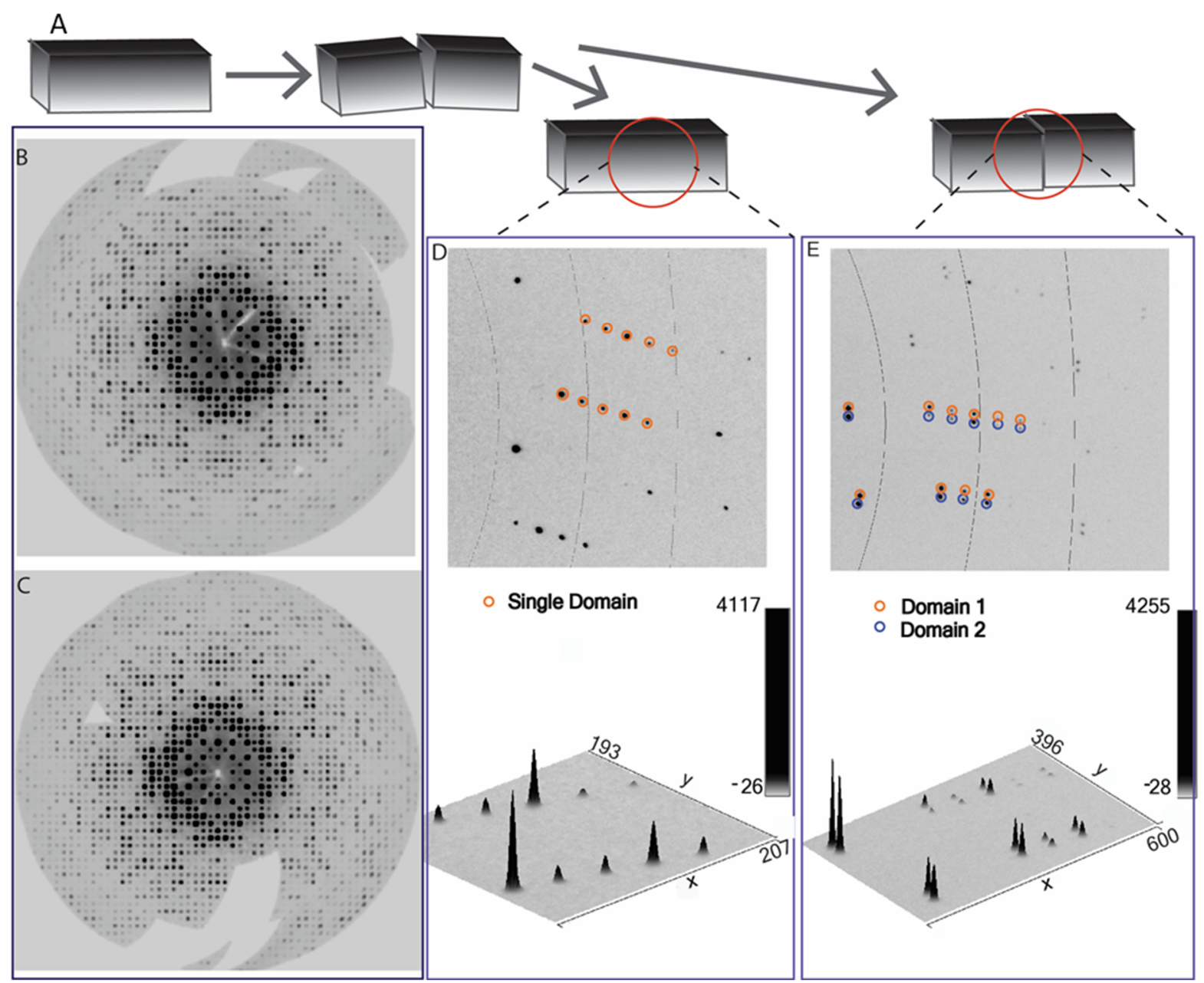

Fig. 3. Crystallographically precise self-healing in crystals of 1. (A) Schematic depiction of self-healing. 2D projection of reconstructed 3D Ewald sphere viewed along $C^{*}$ for a (B) pristine and a (C) neatly-healed crystal. An image showing the measured 2D diffraction pattern (top panel) and its corresponding 3D intensity profile (bottom panel) for (D) neatly-healed crystal and (E) imperfectly-healed crystal with visible crack line.

of the crack line (Fig. 2G-H, Fig.S10), explaining the electrostatic attraction between the two fractured pieces. More importantly, the neatly-healed crystals, though exhibit a small potential, showed no such electrostatic potential gradient across the crack line on the $(100) /(010)$ faces (Fig. S11).

Since crystalline integrity is the single most crucial aspect that critically impacts almost all the physical properties in case of molecular crystals, we examined the self-healing efficiency with respect to the internal order and alignment of rejoined pieces of $\mathbf{1}$ using micro-focus SCXRD technique (beam diameter $=300 \mu \mathrm{m}$ ). The neatly-healed crystals produced remarkably sharp diffraction peaks, comparable to that of the pristine crystals (Fig. 3B, C, D; Fig. S12-13). We found no split-peaks even at higher resolution (Fig. 3C). Further, complete diffraction data provided the excellent fit to the structural model ( $R_{1}=0.027$, Table S3), confirming an exceptionally efficient self-healing with identical crystallographic parameters and precise alignment of domains of broken pieces (see Table S1, S3-S5, Fig. S12, S13). On the other hand, an imperfectly-healed crystal with a visible crack showed sharp but doubly-split diffraction peaks, revealing 
the misalignment of the two crystalline domains (Fig. 3E, Fig. S12). The neatly-healed crystals show $\sim 100 \%$ efficiency in the SCXRD data.

Further, using a custom designed state-of-the-art dark-field Mueller matrix microscopy system (27) (Fig. S14) having a spatial resolution of $\sim 300 \mathrm{~nm}$, we probed the nanoscale order and quantitatively studied the healing efficiency through the microscopic anisotropy property of crystals, including at the crack-junction. Using the Mueller matrix, which encodes the full polarization response of the interacting medium, one can efficiently probe and quantify anisotropic polarizability of crystalline or other ordered materials using suitable combinations of polarization states of light $(28,29)$. The important linear anisotropy parameters in this context are the magnitude of linear retardance $\left(\delta^{L}\right.$, birefringence), the orientation angle of the retarder $(\theta$, birefringence axis) and linear diattenuation $\left(d^{L}\right)(29)$. The $\delta^{L}$ and $d^{L}$ are called quantitative measures of the order parameters.

Pristine crystals, with high degree of local order, showed strong phase and amplitude anisotropy effects, resulting in the highest magnitudes of anisotropic parameters $\left(\delta^{L}=1.52 \pm 0.16\right.$ rad and $\left.d^{L}=0.209 \pm 0.003\right)$ (Fig. 4A-C). The neatly-healed crystals also showed an impressive internal 


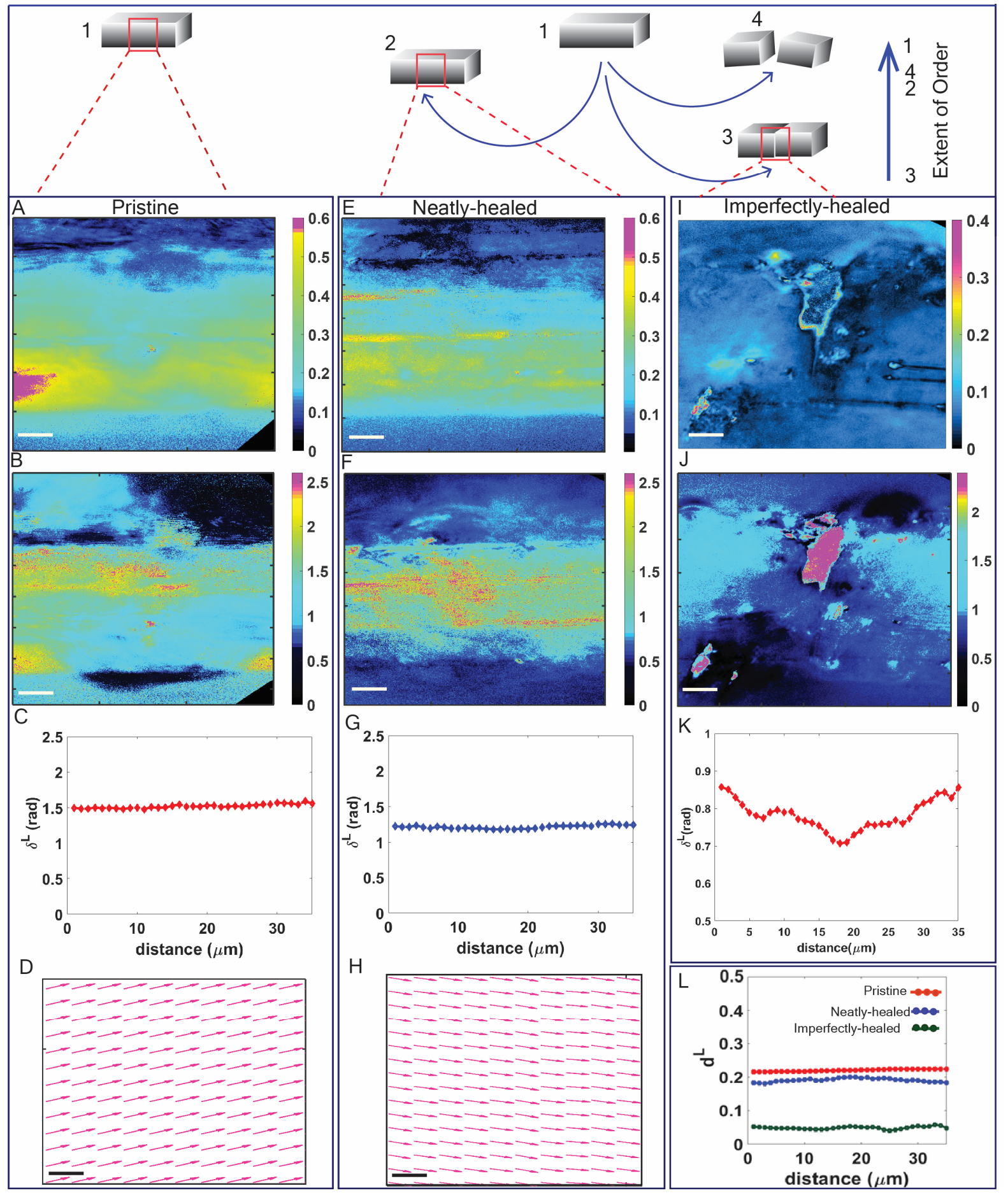

Fig. 4. Quantitative assessment of structural order using Mueller Matrix analysis. (A), $(\mathrm{E})$ and $(\mathrm{I})$ Linear retardance parameter $\left(\delta^{L}\right) ;(\mathrm{C}),(\mathrm{F})$ and $(\mathrm{J})$ Linear diattenuation parameter $\left(d^{L}\right) ;(\mathrm{C}),(\mathrm{G})$ and $(\mathrm{K})$ spatial variation plots of $\delta^{L}$; corresponding to pristine, neatly-healed and imperfectly-healed crystals, respectively. Scale bar is $25 \mu \mathrm{m}$. (L) Comparison of spatial variation plots of $d^{L}$ for different crystals. (D) and $(\mathrm{H})$ The microscopic orientation (uniform along the crystal) of the retarder axis of the pristine and neatly-healed crystal, respectively. Scale bar is $4 \mu \mathrm{m}$. The slight offset in their orientation is due to the manual alignment of crystals on the sample stage. See Fig. S15-18 and Table S6 for detailed MM data. 
order, but found to have slight decrease in parameters $\left(\delta^{L}=1.34 \pm 0.1 \mathrm{rad}\right.$ and $d^{L}=$ $0.189 \pm 0.005)$ (Fig. 4E-G) (Fig S15, S16). This quantifies the self-healing efficiency in the neatly-healed crystals of 1 as $~ 85 \%$. Note that this is in the context of the nanoscale order. Our technique captures any imperfections more precisely than the macroscopic imaging or SCXRD techniques $(15,16)$. Spatially separated fractured pieces (not allowed to rejoin) also showed the order comparable to that of the neatlyhealed crystals (Fig. S18). This suggests that the mechanical impact permanently decreases the overall structural order of the crystals by 10-15\%. This slight decrease in the microscopic orientation of the axis of the retarder (shown by arrow) for the neatly-healed crystals as compared to the pristine crystals is evident from Fig. 4D, $\mathrm{H}$. However, the largely ordered nature of the structure at nanoscale in neatly-healed crystals is clear from our results.

In case of imperfectly-healed crystals with visible cracks, a large decrease in the parameters $\left(\delta^{L}=0.70 \pm 0.01 \mathrm{rad}\right.$ and $\left.d^{L}=0.07 \pm 0.008\right)$ is seen (Fig. $\left.4 \mathrm{I}-\mathrm{K}\right)$, suggesting the disorganization of local anisotropic domains, leading to reduction of net anisotropy effects to $\sim 50 \%$. Note that the nanoscale spatial variation of the order parameters for cracked crystal drops significantly at the crack line or healed junction while for the neatly-healed crystals it did not exhibit any noticeable variations (Fig. 4G, K). This indicates that the neatly healed crystals not only retain overall long- range crystalline order but also successfully heal at the crack line with nano-scale precision, highly required for piezoelectric materials, particularly in precession applications such as transducers.

In conclusion, our novel approach employing piezoelectric property paves the way for truly autonomous self-healing with unprecedented retention of crystallographic order in single crystal based organic materials $(19,30,31)$. Our results demonstrating generation of large electrostatic charges and the unexplored autonomous selfalignment could be a common and inherent phenomenon in certain types of synthetic and natural piezoelectric materials. Combining the piezoelectricity based self-healing mechanisms with other known strategies in polymers, gels and composite materials could allow overcoming the challenge of autonomous recombination (utilizing local electrostatic charge generation), thus attaining new levels of self-healing efficiencies (2-14). In the inherently piezoelectric biological materials, including in bone and tissue repair, the electrostatic charges generated by mechanical impact are known to play a key role in wound healing, following complex mechanisms (1). The insights from our study, having access to atomic resolution structural information from the piezoelectric crystals, could be valuable for the deeper understanding of self-healing in biological materials, such as in bones, muscles, collagen, sub-cell components such as microtubules and cell-walls, molecular level mechanisms of which remain poorly understood.

\section{References and Notes:}

1. D. Kim, S. A. Han, J. H. Kim, J. H. Lee, S. W. Kim, S. W. Lee, Biomolecular Piezoelectric Materials: From Amino Acids to Living Tissues. Adv. Mater. 32, 1906989 (2020). 
2. Y. Yanagisawa, Y. Nan, K. Okuro, T. Aida, Mechanically robust, readily repairable polymers via tailored noncovalent cross-linking. Science. 359, 72-76 (2018).

3. H. Yamagishi, H. Sato, A. Hori, Y. Sato, R. Matsuda, K. Kato, T. Aida, Self-assembly of lattices with high structural complexity from a geometrically simple molecule. Science. 361, 1242-1246(2018).

4. S. Wang, M. W. Urban, Self-healing Polymers. Nat. Rev. Mater. 5, 562-583 (2020).

5. M. W. Urban, D. Davydovich, Y. Yang, T. Demir, Y. Zhang, L. Casabianca, Key-andlock commodity self-healing copolymers. Science. 362, 220-225 (2018).

6. A. Pena-Francesch, H. Jung, M. C. Demirel, M. Sitti, Biosynthetic self-healing materials for soft machines. Nat. Mater. 19, 1230-1235 (2020).

7. P. Cordier, F. Tournilhac, C. Soulié-Ziakovic, L. Leibler, Self-healing and thermoreversible rubber from supramolecular assembly. Nature. 451, 977-980 (2008).

8. M. Burnworth, L. Tang, J. R. Kumpfer, A. J. Duncan, F.L. Beyer, G.L. Fiore, S.J. Rowan, C. Weder, Optically healable supramolecular polymers. Nature, 472, 334-337 (2011).

9. N. Holten-Andersen, M. J. Harrington, H. Birkedal, B. P. Lee, P. B. Messersmith, K. Y. C. Lee, J. H. Waite, pH-induced metal-ligand cross-links inspired by mussel yield selfhealing polymer networks with near-covalent elastic moduli. Proc. Natl. Acad. Sci. U.S.A. 108, 2651-2655 (2011).

10. Q. Zhang, C. Y. Shi, D. H. Qu, Y. T. Long, B. L. Feringa, H. Tian, Exploring a naturally tailored small molecule for stretchable, self-healing, and adhesive supramolecular polymers. Sci. $a d v$. 4, eaat8192 (2018).

11. C. H. Li, C. Wang, C. Keplinger, J. L. Zuo, L. Jin, Y. Sun, P. Zheng, Y. Cao, F. Lissel, C. Linder, X. Z. You, Z. Bao, A highly stretchable autonomous self-healing elastomer. Nat. Chem. 8, 618-624 (2016).

12. S. R. White, N. R. Sottos, P. H. Geubelle, J. S. Moore, M. R. Kessler, S. R. Sriram, E. N. Brown, S. Viswanathan, Autonomic healing of polymer composites. Nature. 409, 794797 (2001).

13. H. Ying, Y. Zhang, J. Cheng, Dynamic urea bond for the design of reversible and selfhealing polymers. Nat. Commun. 5, 3218(2014).

14. X. Chen, M. A. Dam, K. Ono, A. Mal, H. Shen, S. R. Nutt, K. Sheran, F. Wudl, A thermally re-mendable cross-linked polymeric material. Science. 295, 1698-1702 (2002).

15. L. Zhang, J. B. Bailey, R. H. Subramanian, A. Groisman, F. A. Tezcan, Hyperexpandable, self-healing macromolecular crystals with integrated polymer networks. Nature. 557, 86-91 (2018).

16. P. Commins, M. B. Al-Handawi, D. P. Karothu, G. Raj, P. Naumov, Efficiently selfhealing boronic ester crystals. Chem. Sci. 11, 2606-2613 (2020).

17. S. K., Karan, R. Bera, S. Paria, A. K. Das, S. Maiti, A. Maitra, B. B. Khatua, An approach to design highly durable piezoelectric nanogenerator based on self-poled 
PVDF/AlO-rGO flexible nanocomposite with high power density and energy conversion efficiency. Adv. Energy Mater. 6, 1601016 (2016).

18. S. Das, A. Mondal, C. M. Reddy, Harnessing molecular rotations in plastic crystals: a holistic view for crystal engineering of adaptive soft materials. Chem. Soc. Rev. 49, 8878 $-8896(2020)$.

19. S. Saha, M. K. Mishra, C. M. Reddy, G. R. Desiraju, From molecules to interactions to crystal engineering: mechanical properties of organic solids. Acc. Chem. Res. 51, 29572967 (2018).

20. P. Naumov, S. Chizhik, M.K. Panda, N.K. Nath, E. Boldyreva, Mechanically responsive molecular crystals. Chem. Rev. 115, 12440-12490 (2015).

21. J. Harada, T. Shimojo, H. Oyamaguchi, H. Hasegawa, Y. Takahashi, K. Satomi, Y. Suzuki, J. Kawamata, T. Inabe. Directionally tunable and mechanically deformable ferroelectric crystals from rotating polar globular ionic molecules. Nat. Chem. 8, 946-952 (2016).

22. S. Varughese, M. S. R. N. Kiran, U. Ramamurty, G. R. Desiraju, Nanoindentation in crystal engineering: quantifying mechanical properties of molecular crystals. Angew. Chem. Intl. Ed. 52, 2701-2712 (2013).

23. H. Y. Ye, Y. Y. Tang, P. F. Li, W. Q. Liao, J. X. Gao, X. N. Hua, H. Cai, P. P. Shi, Y. M. You, R. G. Xiong, Metal-free three-dimensional perovskite ferroelectrics. Science. 361, 151-155 (2018).

24. D. J. Ellison, B. Lee, V. Podzorov, C. D. Frisbie, Surface potential mapping of SAMfunctionalized organic semiconductors by Kelvin probe force microscopy. Adv. Mater. 23, 502-507 (2011).

25. S. Guerin, A. Stapleton, D. Chovan, R. Mouras, M. Gleeson, C. McKeown, M. R. Noor, C. Silien, F. M. F. Rhen, A. L. Kholkin, N. Liu, T. Soulimane, S. A. M. Tofail, D. Thompson, Control of piezoelectricity in amino acids by supramolecular packing. Nat. Mater. 17, 180-186 (2018).

26. K. Kubota, Y. Pang, A. Miura, H. Ito, Redox reactions of small organic molecules using ball milling and piezoelectric materials. Science, 366, 1500-1504 (2019).

27. S. Chandel, J. Soni, S. Kumar Ray, A. Das, A. Ghosh, S. Raj, N. Ghosh, Complete polarization characterization of single plasmonic nanoparticle enabled by a novel Darkfield Mueller matrix spectroscopy system. Sci. Rep. 6, 26466 (2016).

28. D. H. Goldstein, Polarized Light (CRC Press, 2016).

29. S. K. Ray, S. Chandel, A.K. Singh, A. Kumar, A. Mandal, S. Misra, P. Mitra, N. Ghosh, Polarization-tailored Fano interference in plasmonic crystals: A Mueller matrix model of anisotropic Fano resonance. ACS nano, 11, 1641-1648 (2017).

30. G. R. Desiraju, J. J. Vittal, A. Ramanan, Crystal engineering: a textbook. (World Scientific, 2011).

31. J. M. McCracken, B. R. Donovan, T. J. White, Materials as Machines. Adv. Mater. 32 , 1906564 (2020). 
Acknowledgments: We acknowledge Prof. Damien Thompson of University of Limerick for his valuable inputs and suggestions. Micro and Nano Characterization Facility (MNCF) at the Centre for Nano Science and Engineering (CeNSE), Indian Institute of Science, Bangalore, India is acknowledged for KPFM measurement. We thank Mr. Kishore Kumar from Toshniwal Brothers (SR) Pvt. Ltd., Bangalore - 560094 for PFM measurement.

Funding: CMR thanks DST, New Delhi for Swarnajayanti Fellowship (DST/SJF/CSA-02/2014-15) and SERB for funding (No: EMR/2017/005008). SB thanks DST-INSPIRE for Senior Research Fellowship.

Author contributions: Mechanical manipulation of crystals was done by SB and SuD. SCXRD was handled by SoD and SB. Material was synthesized by RC, SM, AM and SB. NI, SEM were performed by SB. MMM was performed by SC, AT, NK under the supervision of NG. Piezoelectric measurement was done by SKK under the supervision of BBK. KPFM was done by SB and SuD. All the experiments were planned by SB and CMR. CMR supervised the whole project. SB and CMR analyzed all the results and co-wrote the manuscript taking inputs from all coauthors. Competing interests: Authors declare no competing interests; Data and materials availability: All data is available in the main text or the supplementary materials.

\section{Supplementary Materials:}

Materials and Methods

Figures S1-S21

Tables S1-S6 


\section{Materials and Methods}

\section{Materials:}

Synthesis and crystallization: $3,3^{\prime}, 5,5^{\prime}$-Tetramethyl-4,4'-bipyrazole was synthesized by previously reported procedure (32). To prepare it, reagents (acetylacetone, sodium hydride, iodine) brought from SigmaAldrich were used as it is without further purification.

After characterizing the compound with NMR, $100 \mathrm{mg}$ of the product is added to $40 \mathrm{~mL}$ of DMSO in a nicely cleaned, dust free conical flask. Thus, the prepared solution is boiled for 10 minutes on a hot plate. After that the solution is kept for 2030 days to obtain nice needle shaped single crystals of 1 .

\section{Methods:}

Single crystal X-ray diffraction (SCXRD): X-ray diffraction data for the crystal 1 was collected at $100 \mathrm{~K}$ on a SuperNova, Eos diffractometer using monochromatic $\mathrm{Cu}$-Ka radiation having a $300 \mu \mathrm{m}$ beam size. Using Olex2, (1.2.9 version) (33) the structure was solved with the SHELXT structure solution program using intrinsic phasing algorithom and refined with the ShelXL refinement package using Least Squares minimization (34). Displacement parameters of all non-hydrogen atoms were refined anisotropic. All the crystal packing diagrams were prepared using Mercury (3.10.1 version) and Chimera software.

Powder XRD: The PXRD patterns were collected on a Rigaku SmartLab with a $\mathrm{Cu}$ $\mathrm{Ka}$ radiation. The tube voltage and amperage were set at $40 \mathrm{kV}$ and $50 \mathrm{~mA}$ respectively. Sample was scanned between 5 and $40^{\circ} 2 \theta$ with a step size of $0.02^{\circ}$. The instrument was previously calibrated using a silicon standard.

Scanning Electron Microscopy (SEM): The images were obtained using Zeiss DSM 950 and FEI QUANTA 200 3D microscope operating at $10 \mathrm{kV}$ using tungsten filament as the electron S4 source. Before the imaging, the samples were sputtered with gold (nano-sized film) using an SCD 040 Balzers Union sputterer to avoid charging during SEM analyses.

Kelvin Probe Force Microscopy (KPFM):

KPFM is a noncontact technique that measures the local nanoscale surface potential and topography, directly correlating the two. Measurements were performed by Bruker Dimension Icon AFM instrument with Pt/lr coated tip [SCM-PIT-V2] with tip radius of $25 \mathrm{~nm}$, resonant frequency of $70 \mathrm{kHz}$ and force constant $\mathrm{k}$ of $3-4 \mathrm{~N} \mathrm{~m}^{-1}$. All KPFM scans were conducted with $\left(V_{\mathrm{AC}}=500 \mathrm{mV}\right)$ and lift height $(d=30 \mathrm{~nm})$. The topographic scan was performed by employing tapping mode. The spatial resolution of KPFM is around $25 \mathrm{~nm}$. Work function of the tip is $\sim 4.9 \mathrm{eV}$.

Piezoresponse Force Microscopy (PFM): The PFM measures the mechanical response when an electrical voltage is applied to the sample surface with a conductive tip of an AFM. In response to the electrical stimulus, the sample then locally expands or contracts. Instrument used is MFP-3D scanning probe microscope (SPM) system (Asylum Research, CA, USA). Topographic images, amplitude and phase images of the samples were obtained and analysed by DART (Dual AC Resonance Tracking) PFM mode which is a scanning technique used in PFM that allows dual excitation of the cantilever to independently measure both the amplitude 
and resonance frequency of the cantilever, improving spatial resolution and sensitivity. The AFM imaging and spectroscopy technique used ASYELEC-01 silicon conductive cantilever probes. Vertical PFM (VPFM) was explored to measure out-ofplane polarization $\left(d_{z z}\right)$ by recording the tip deflection signal at the frequency of modulation. And switching Spectroscopy obtained by DART spectroscopy mode.

Nanoindentation: Nanoindentation experiments have been performed on the all non-equivalent faces of single crystals using the TI Premier from Hysitron, Minneapolis, USA, equipped with an in-situ Scanning Probe Microscope (SPM). A Berkovich tip (three-sided pyramidal tip with a total included plane-edge angle of $142.3^{\circ}$ ) of radius $\sim 150 \mathrm{~nm}$ was employed to determine the hardness $(H)$ and elastic modulus $(E)$ of the crystals. The $H$ and $E$ were extracted using the standard OliverPharr (O\&P) method. The complete details of the method can be found elsewhere (35).

DSC-TGA: DSC experiments were conducted on a Mettler-Toledo DSI1 STAR instrument on accurately weighed samples $(4-6 \mathrm{mg})$ placed in hermetically sealed aluminium crucibles $(40 \mu \mathrm{L})$ upon scanning in the range of $30^{\circ} \mathrm{C}$ to $350^{\circ} \mathrm{C}$ at a heating rate of $5^{\circ} \mathrm{C} / \mathrm{min}$ under a dry nitrogen atmosphere (flow rate $80 \mathrm{~mL} / \mathrm{min}$ ).

TGA was performed on a Mettler-Toledo TGA/SDTA $851^{\mathrm{e}}$ instrument. Approximately $6-13 \mathrm{mg}$ of the sample was added to an aluminium crucible and heated from 30 to $350^{\circ} \mathrm{C}$ at a rate of $5^{\circ} \mathrm{C} / \mathrm{min}$.

Mechanical manipulation under optical microscopy equipped with a highspeed camera: Mechanical manipulation of the single crystals was performed with the help of a pair of forceps and needle head under Leica (M205 FCA) polarized optical microscope equipped with Fastcam Mini high-speed camera.

\section{Dark field Mueller Matrix Microscopy (MMM):}

We have used a custom designed dark field polarization spectroscopic microscopy system capable of recording the complete Mueller matrix of a sample with great precision $(27,29,38)$. The system has the exceptional ability to record spatial maps (images) of the Mueller matrix elements for a selected wavelength with spatial resolution down to $~ 300 \mathrm{~nm}(27,29,38)$. This Mueller matrix system, integrated with a dark field microscope, employs excitation with broadband light and allowed subsequent recording of sixteen polarization resolved images (at a selected wavelength between $=400-725 \mathrm{~nm}$ ) by sequential generation and analysis of four optimized elliptical polarization states using a polarization state generator (PSG) and polarization state analyzer (PSA) unit, respectively $(27,29,38)$. The high spatial resolution of the polarization resolved Mueller matrix elements are achieved by using high numerical aperture (NA) condenser and objective in the dark field microscopic arrangement, where the outstanding issues related to the polarization response of the high NA microscopic geometry is taken care of by a robust eigenvalue calibration method $(27,29,38)$.

\section{Supplementary Text}

\section{Calculation of healing time:}

A video during mechanical damage and self-healing process for a crystal was recorded at 1000 frames per second. Therefore, each frame is related to $1 / 1000$ second or $1 \mathrm{~ms}$ event. Then each frame of the video was analyzed to check the mechanical damage and self-healing events, frame by frame. During the healing event we observed a difference of just one frame between a crack visibility and 
disappearance events. Hence the healing duration is taken as $\sim 1 \mathrm{~ms}$ (or less). It is noteworthy to mention that due to a lack of sufficient light source in our facility, the video could not be recorded beyond $1000 \mathrm{fps}$, hence the evaluation of healing time could not be done with better precision than this.

2. Piezoelectric coefficient estimation: Random orientation of the crystals in pellets, made using microcrystalline bulk samples, leads to contribution of different faces of the crystals during the $d_{33}$ measurements, resulting in a combined response that varies with crystal faces. Because of the degree of randomness in the bulk sample, different piezoelectric constants may contribute to the measured piezoelectric response, exhibiting higher piezoelectric constant than that measured by PFM on only the side face of a single crystal. It is noteworthy to mention that PFM measurement on the top face of the needle shape crystal was not possible due to sample mounting limitations. It is quite difficult to prepare exact pellet with same orientation of the microcrystals for each samples due limits of alignment during pellet formation $(36,37)$.

\section{KPFM experiments on cleaved $(001) /(00-1)$ face of the crystal:}

As it was not possible to mount the thin acicular crystals of 1 vertically with the freshly created $(001) /(00-1)$ face, we embedded the crystal in a polymer matrix and cut it perpendicular to $(100) /(010)$ faces to extremely thin slices using a blade, just before the experiment and mounted on the KPFM stage using a silver paste. The experiment was done on the freshly broken top face of the crystal and the first data was collected in about 15 minutes from the time of mechanical breakage.

\section{X-ray diffraction studies on the self-healing crystals:}

Single crystal $\mathrm{X}$-ray diffraction experiments were performed at $T=100 \mathrm{~K}$ on a pristine crystal of 1. Another pristine crystal was taken and broken perpendicular to the long needle axis and then allowed to heal (neatly-healed crystal). Single crystal $\mathrm{X}$-ray diffraction experiment was performed on this crystal at $T=100 \mathrm{~K}$. Diffraction pattern exhibited neither any sign of polycrystalline powder rings nor double peaks close to each other, confirming that the crystal is completely healed with excellent long-range order. Remarkably, at higher resolution, the peaks corresponding to $K \alpha_{1}$ and $K \alpha_{2}$ could be resolved, reflecting the excellent crystalline quality of the healed crystal (Fig S13). The structural model, from a complete data set obtained using a neatly-healed crystal, yielded excellent fit to the diffraction data (Table S3). Both structural models of pristine and healed crystal produced nearly equal statistical parameters for refinement (Table S2). Covalent bond distances and angles (Table S4, S5) including hydrogen bonding conformations (Table S1) for both the models were equal within three times their standard uncertainties.

In the next step, a crystal was broken into two pieces. The pieces were not allowed to self-heal independently. Instead, they were brought close to each other using a pair of forceps at slightly oblique orientation. The crystal now healed exhibits a crack. This imperfectly-healed crystal was centered in such a way that the crack lies at the center of the incident X-ray beam and diffraction images were collected. The diffraction pattern now consists of double peaks close to each other indicative of two domains of the partially healed crystal (Fig. S12). The orientation matrices corresponding to the two domains were determined independently and $94 \%$ of the peaks could be indexed within two tetragonal lattices $[a=b=23.266$ (9) $\AA$ and $c=$ 8.709(3) Å].

The tilt between the domains was determined to be $2.2507^{\circ}, 2.2508^{\circ}$ and $2.2236^{\circ}$ around [100], [010] and [001] directions corresponding to the first domain respectively. The overall tilt between the domains is $2.2321^{\circ}$. 


\section{Dark field Mueller Matrix Microscopy (MMM):}

Mueller matrix images were recorded from the parent/pristine crystal, the healed crystal after mechanical deformation and separately from a cracked crystal. The 4 optimized polarized states controlled by the PSG unit is transmitted on the crystal and the pure scattered light from the crystal is being analyzed (4 polarized state) via the PSA unit. The 16 collected polarized intensities are being combined to formulate the $4 \times 4$ Mueller matrix. Once recorded, the Mueller matrix is subjected to inverse analysis using differential Mueller matrix decomposition, the details of which can be found in our previous publications $(38)$ and elsewhere $(39,40)$. This approach decouples the different polarimetry effects from the composite signals of the measured Mueller matrix elements and accurately quantifies the intrinsic sample polarization anisotropy parameters and their parametric spatial maps. The two linear anisotropy parameters that are important in the context of probing the organization and order of the self-healing crystals are the magnitude of linear retardance $\left(\delta^{L}\right)$ and the orientation angle of the retarder $(\theta)$ and linear diattenuation $\left(d^{L}\right)$. Mueller matrix images were recorded from the pristine crystal, the neatly-healed crystal after mechanical deformation, separately from an imperfectly-healed crystal and fracturedseparated crystals. Please note, Mueller matrix efficiently probe and quantify anisotropic polarizability of crystalline or other ordered materials using suitable combination of such polarization states of light. The optical response of such anisotropic material polarizability is manifested as difference in refractive indices between orthogonal polarization states (27, 39-41).). Specifically, linear anisotropies, namely, linear birefringence (retardance) and linear diattenuation (dichroism) polarization metrics are widely used to probe and quantify the order and organization of crystalline matter $(27,39-41)$.). 


\section{Supporting Figures:}

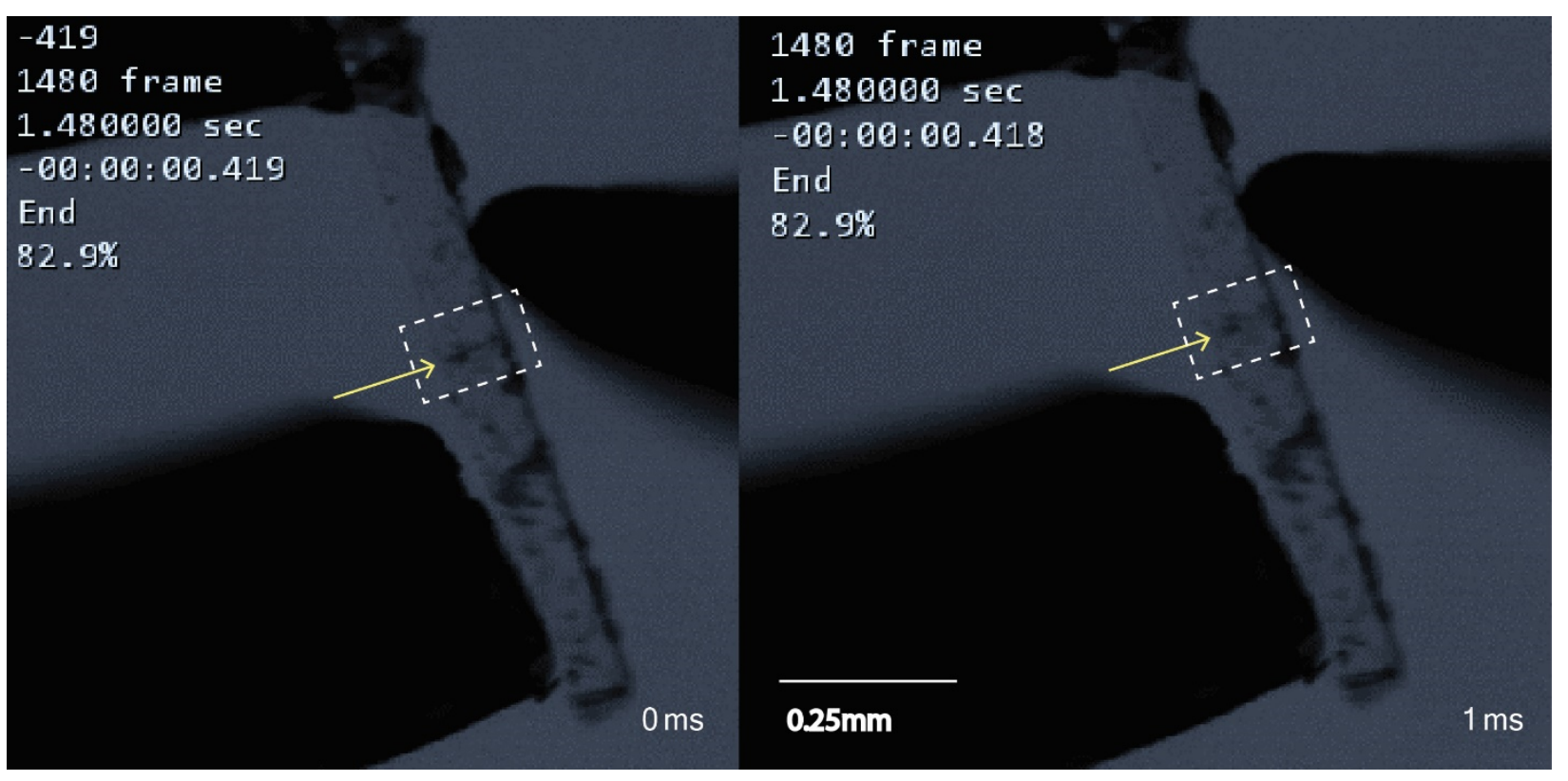

Fig. S1. Calculation of self-healing time. The video was taken at 1000 frames per second. So each frame is related to $1 \mathrm{~ms}$ events. Here two consecutive frames are shown to measure the time taken for a crack healing event. The left frame is showing the crack line (marked by white dotted box and yellow arrow) and the right frame showing the disappeared crack. As the time difference between these two consecutive frames is $1 \mathrm{~ms}$, the time taken for the crack healing is taken approximately as $1 \mathrm{~ms}$. 


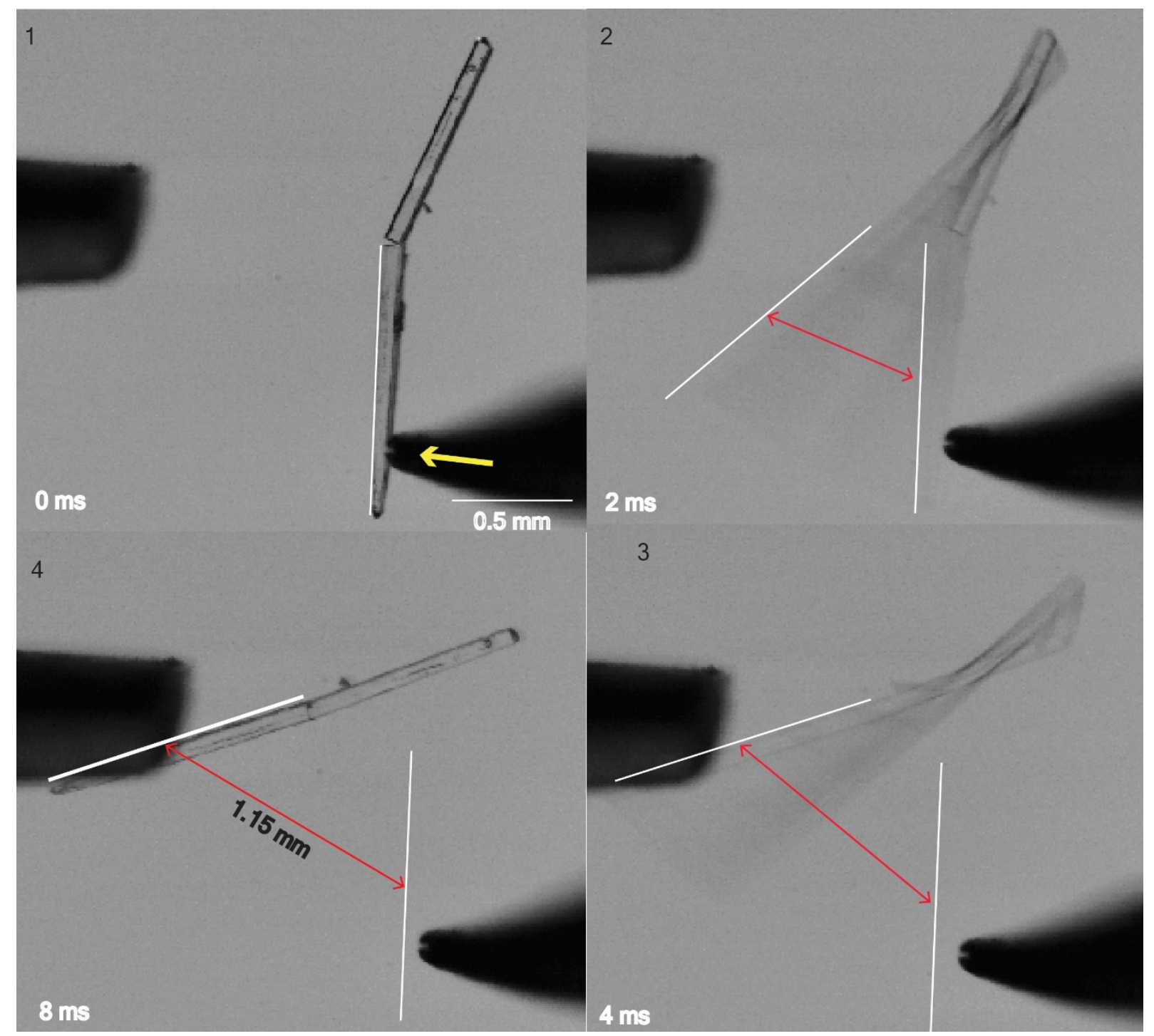

Fig. S2. Mechanical actuation led recombination of fractured crystals via butterfly wing-like motion. 1-4) Videograbs, collected at $500 \mathrm{fps}$, show an event of mechanical actuation followed by recombination covered within $8 \mathrm{~ms}$. This process happens so fast that it looks like a wing movement of honey bees even when recorded at 500 fps. 


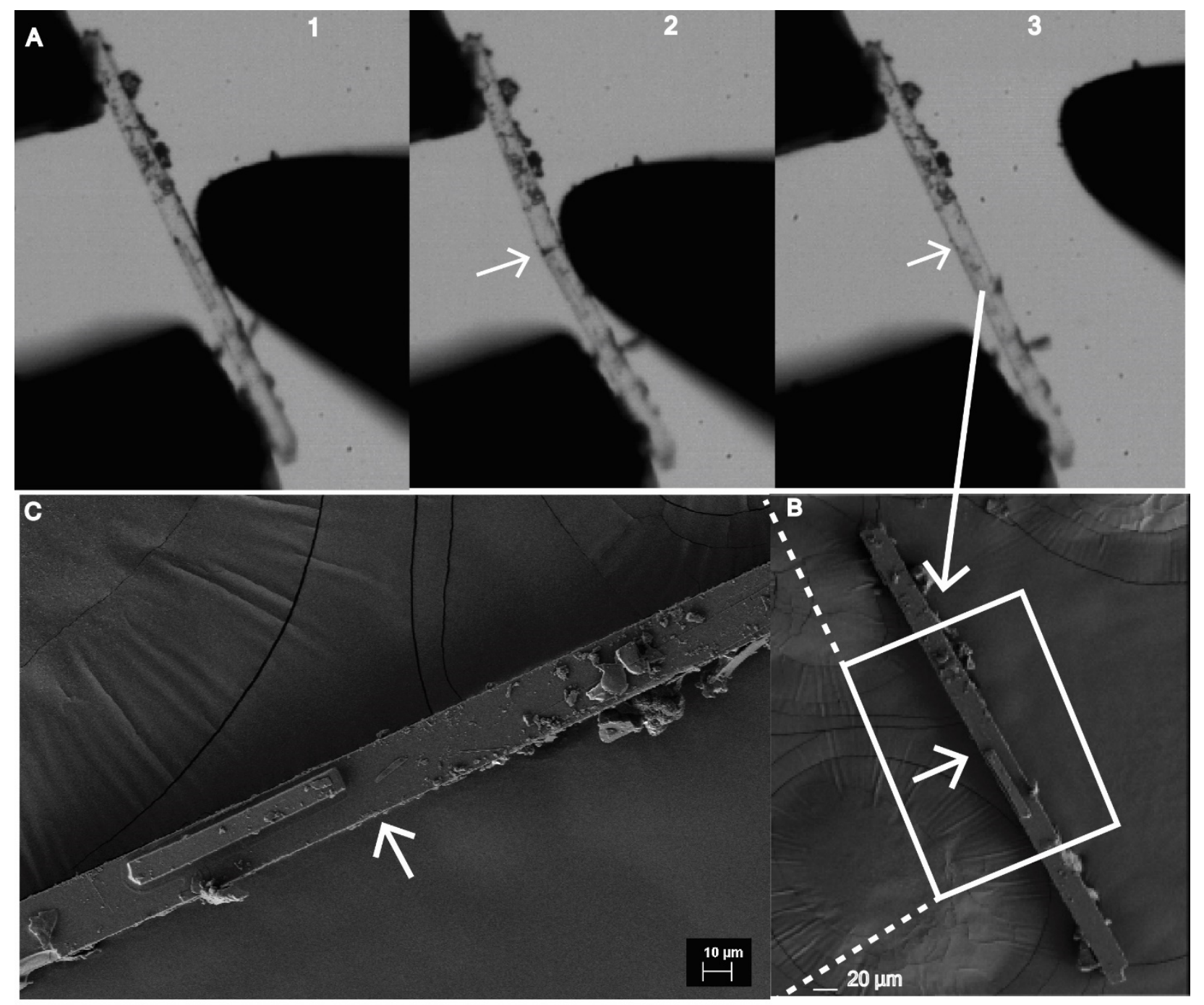

Fig. S3. Examination of self-healing regions by SEM. (A) Video grabs showing the fracture followed by self-healing of a single crystal of 1, which was used for SEM imaging afterwards. B-C) SEM images showing absence of any crack in neatlyhealed crystal (arrows indicate the location of crack-line, which existed prior to healing). 

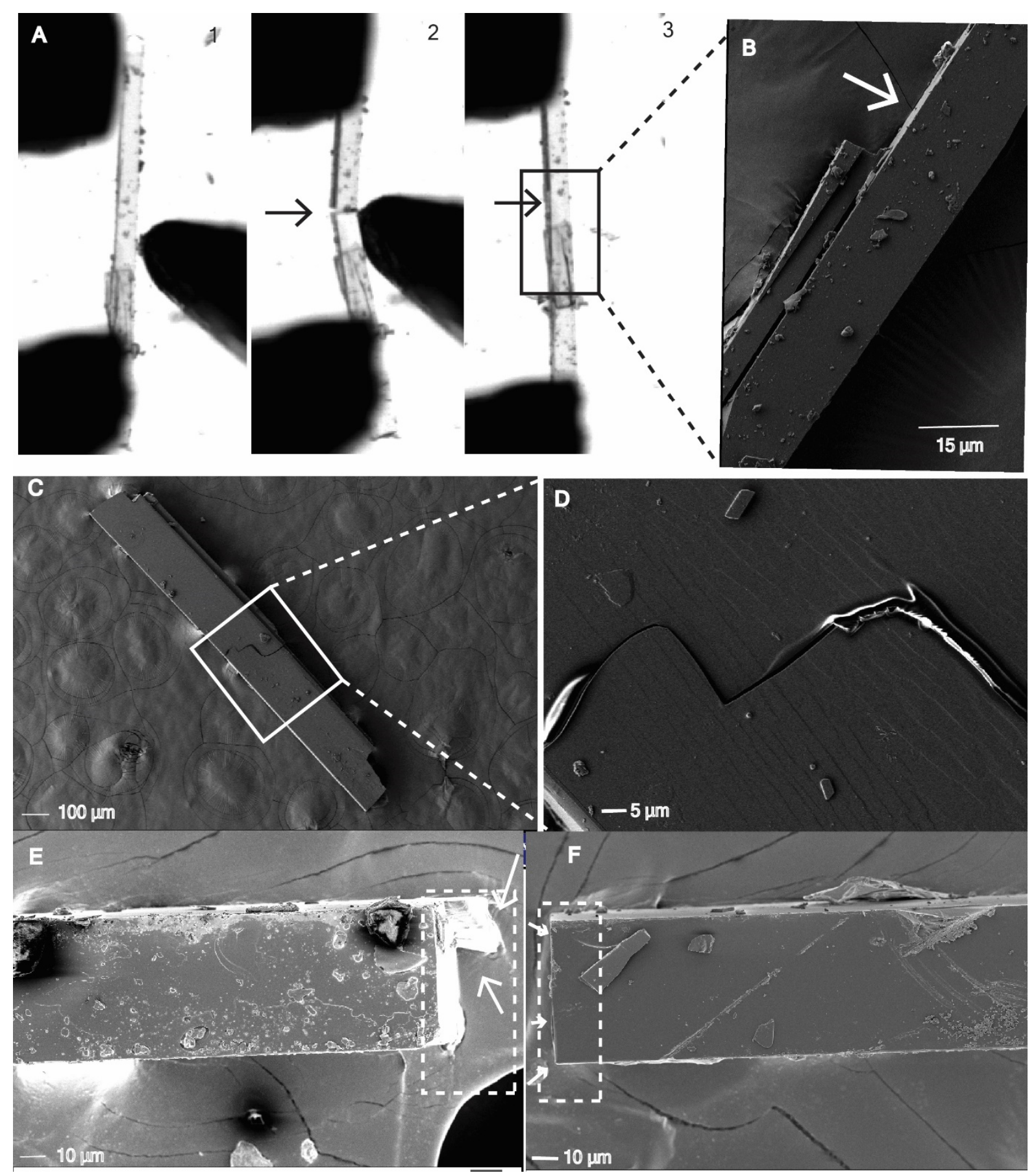

Fig. S4. SEM images of different types of fractured single crystals of 1. A) Video grabs of the healing event of another single crystal which was used for SEM imaging after healing. B) SEM image, showing absence of any crack, confirming efficient healing. Arrows indicate the area where crack was generated. C) SEM image of an imperfectly healed crystals showing a zig-zag crack and D) its magnified version. E) Uneven cleaved face with presence of debrises that abstruck the alignment of domains and hindering complete healing. F) A cleaved crystal edge from a linear crack showing a clean-edge, which generally allows neat-healing. 


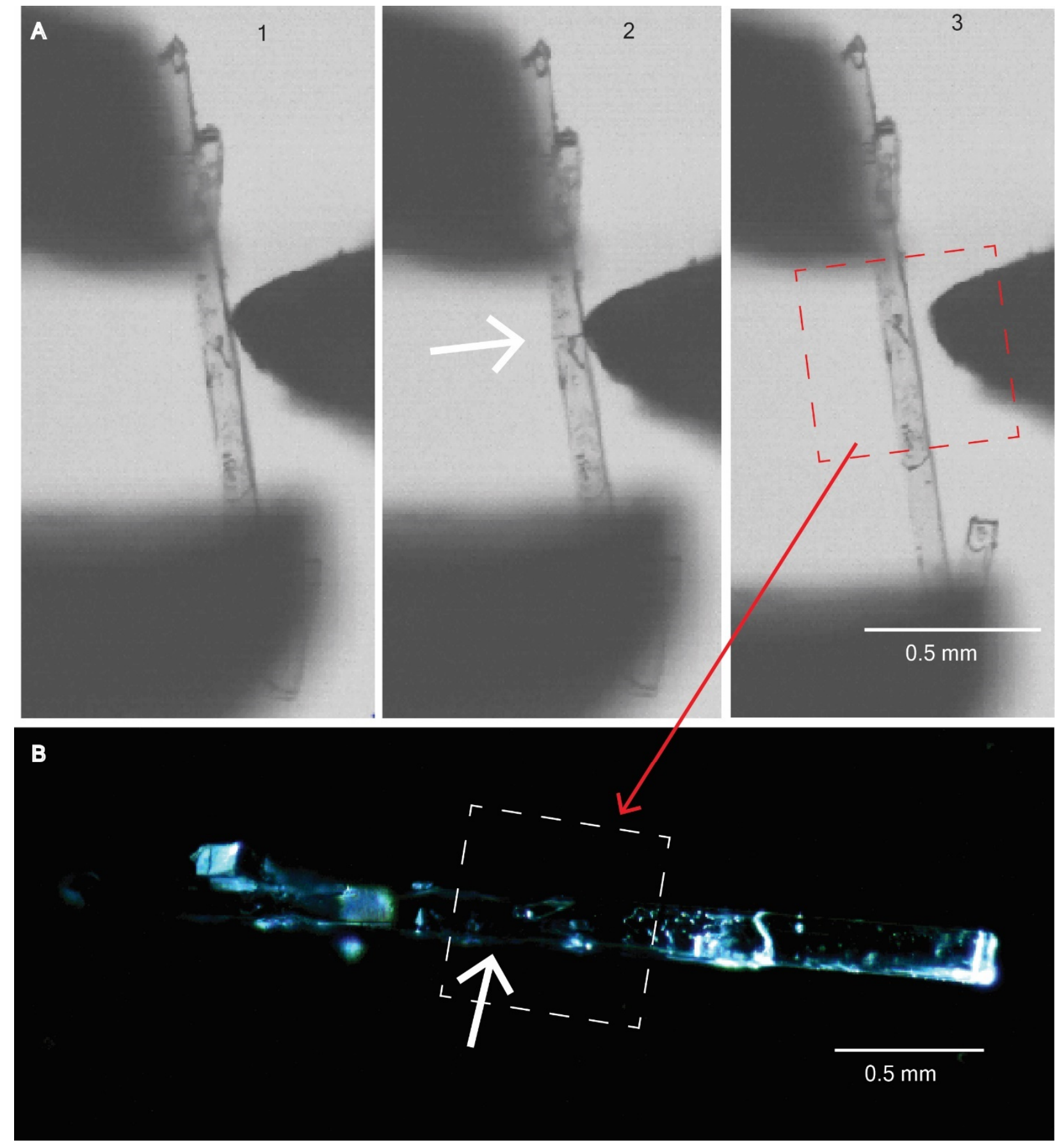

Fig.S5. Examination of self-healing regions by dark-field imaging. (A) Video grabs showing the fracture followed by self-healing of a single crystal of 1 , which was used for dark-field imaging afterwards. B) Dark-field image showing absence of any crack in neatly-healed crystal (arrows indicate the location of crack-line, which existed prior to healing). 


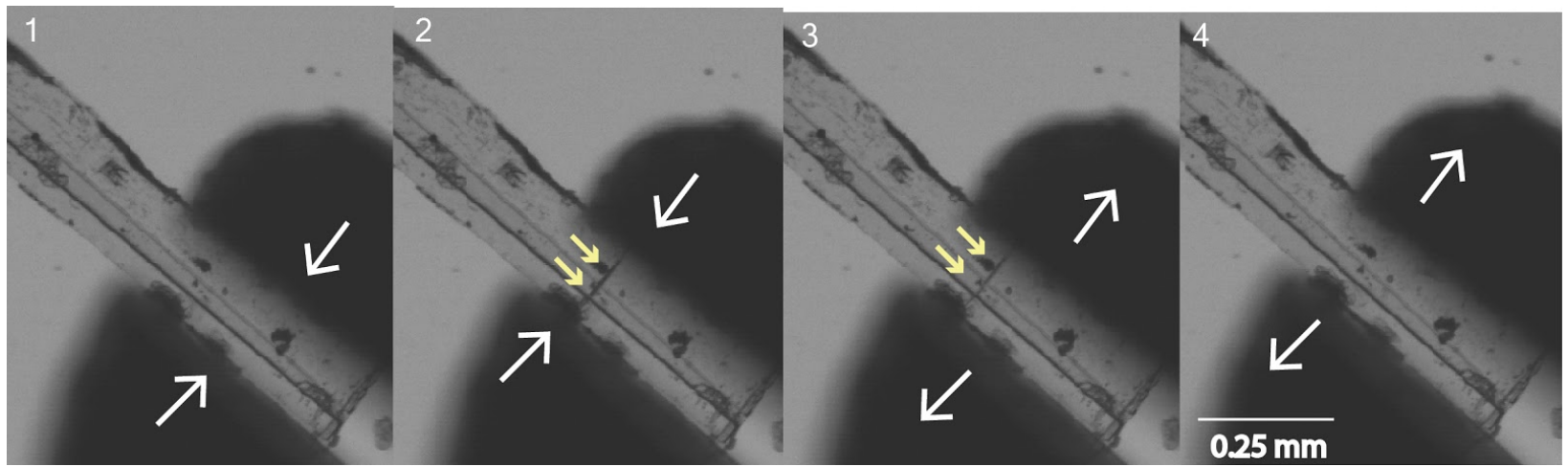

Fig. S6. Crack-healing. Videograbs of instantaneous healing of millimetre length scale cracks, generated by a uniaxial compression of a crystal of 1 using a pair of forceps. 

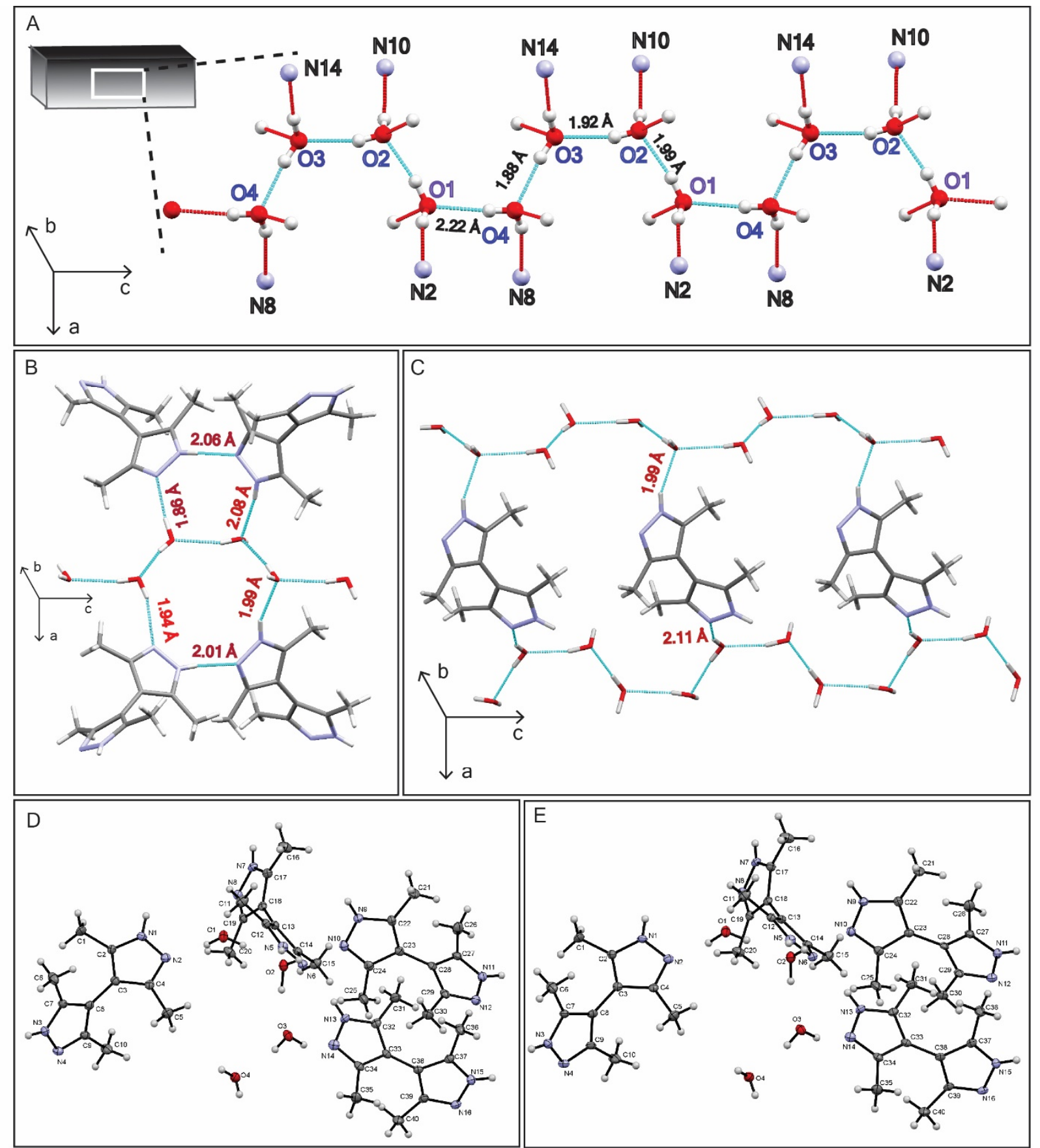

Fig. S7. Packing diagrams of crystal 1. A) One dimensional (1D) cooperative hydrogenbonding of water molecules. Nitrogen: blue; $\mathrm{H}$ bonds: dashed sky-blue lines. B) Hydrogen bonding interaction of bipyrazole molecules with the water chain. C) Parallel water chains are connected by bipyrazole molecules via hydrogen bonding. D \& E) ORTEP diagram (ellipsoids cut at $50 \%$ probability level) of the asymmetric unit for pristine and neatly-healed crystals of 1. 
A

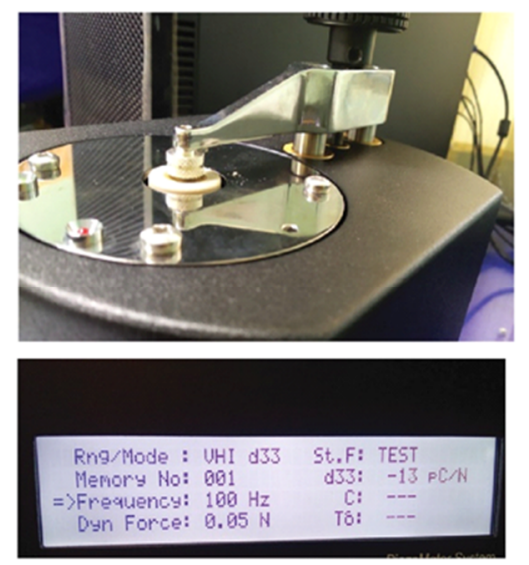

B

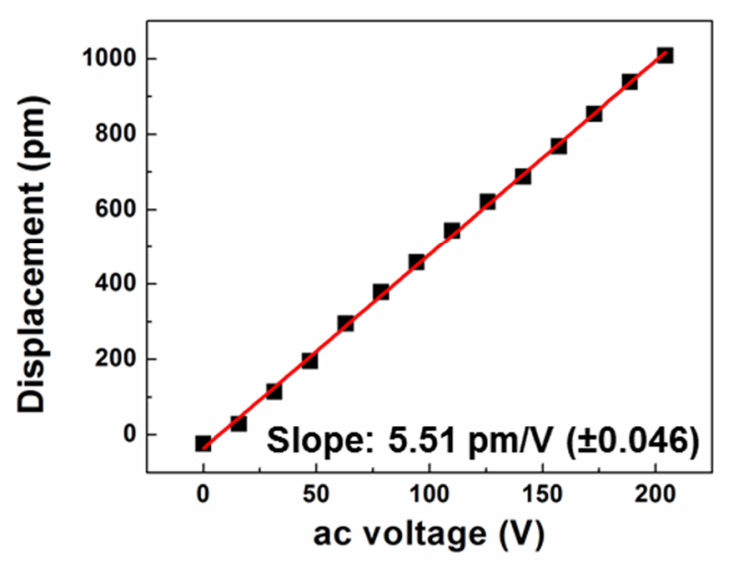

Fig. S8. A) The measurement of $d_{33}$ using piezometer of a bulk powder sample (micro-organic crystal) displaying the value of $-13 \mathrm{pC} / \mathrm{N}$. B) Quantification of the vertical piezoelectric response by calculating the slope of the linear plot of output voltage vs. applied voltage.

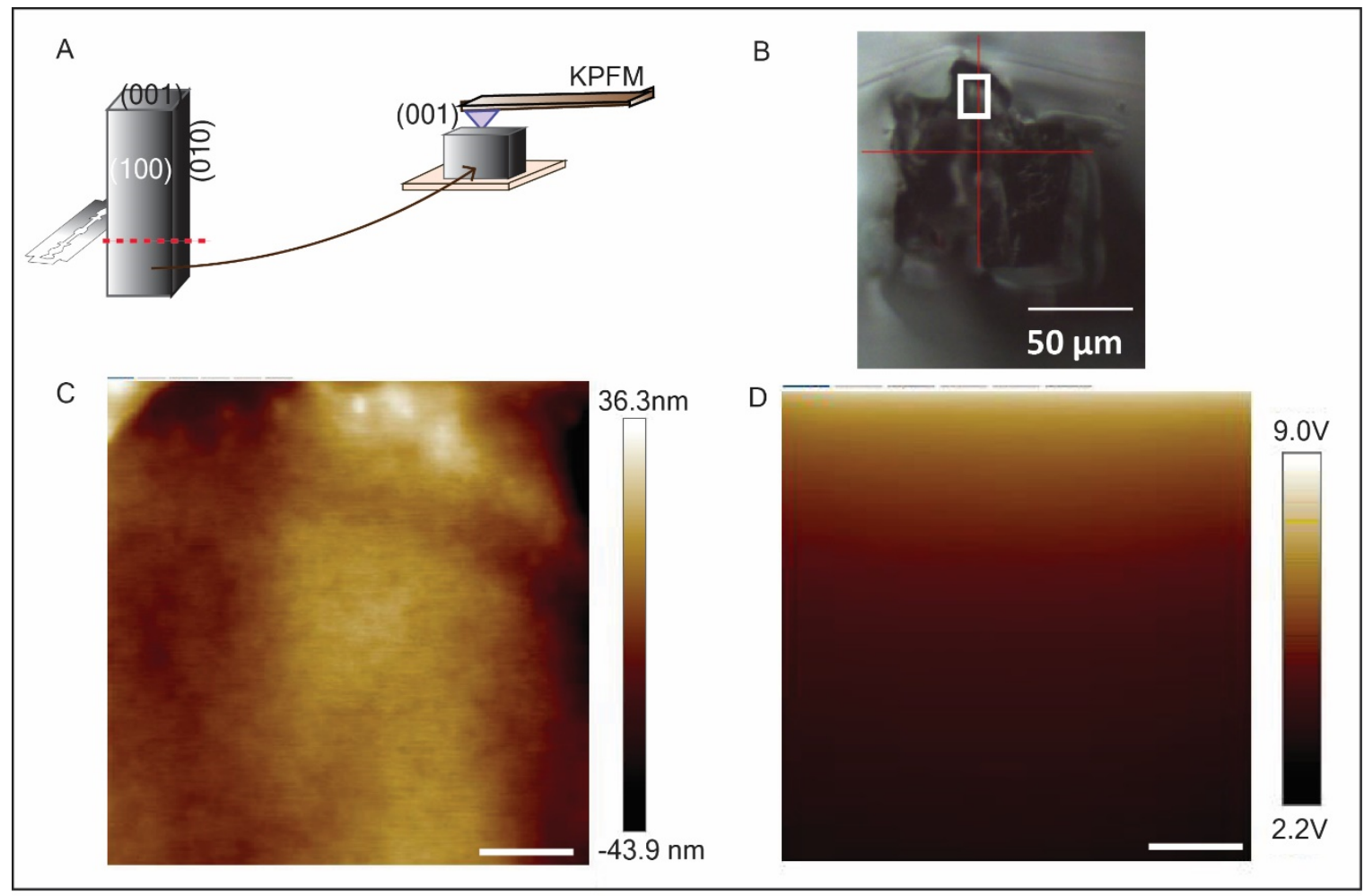

Fig. S9. KPFM on freshly created (001) face. A) Sample preparation for the experiment; B) Optical image of the sample used for the experiment; C) AFM surface topography; (D) KPFM surface potential showing an average surface potential of 4.7 V. Scale bar for $\mathrm{C}$ and $\mathrm{D}$ is $1 \mu \mathrm{m}$. 


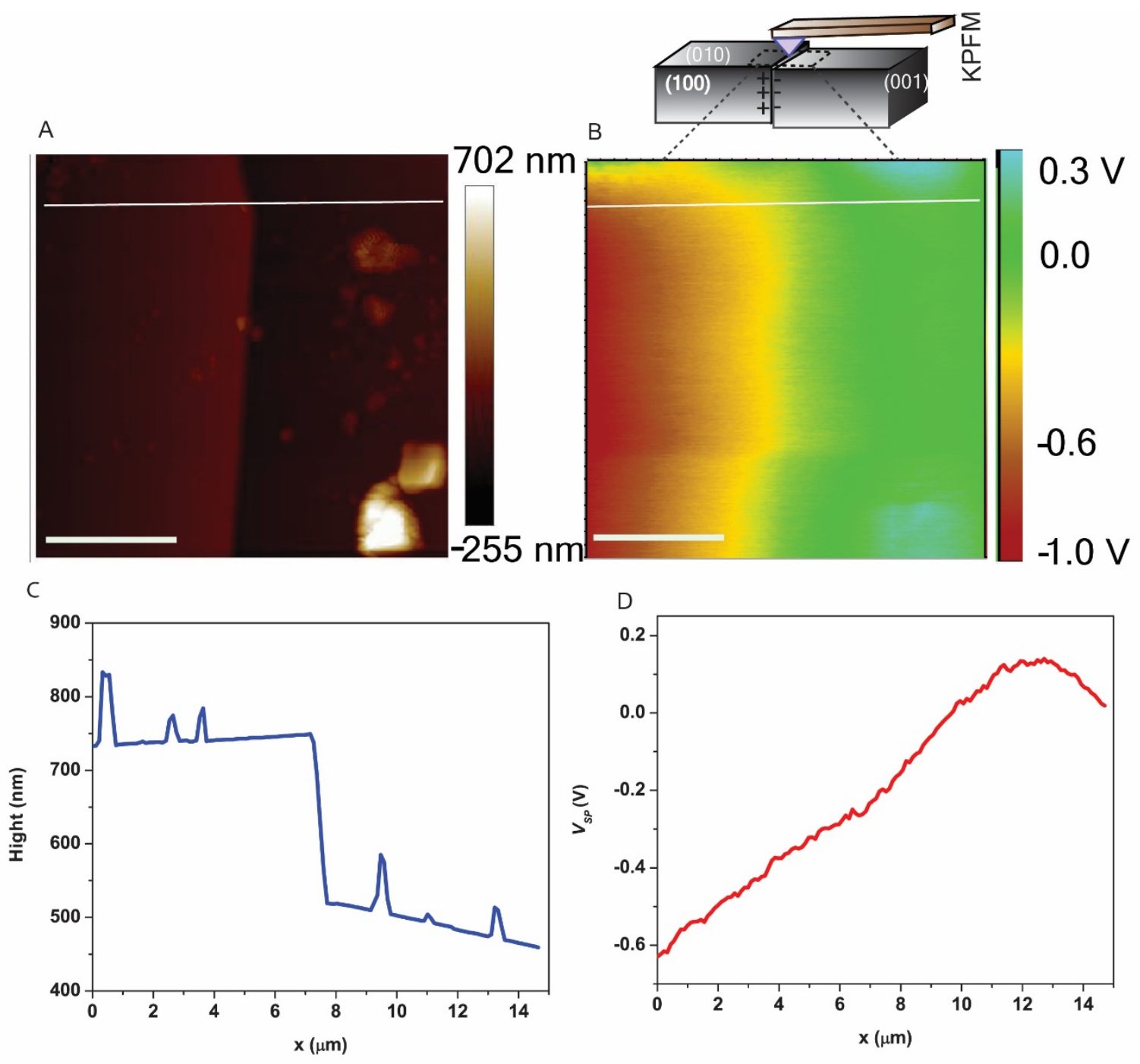

Fig. S10. KPFM on the crack line of an imperfectly-healed crystal. A) AFM surface topography; (B) KPFM surface potential; (C) profiles of height and (D) surface potential across the crack line. Scale bar for $C$ and $D$ is $5 \mu m$. 

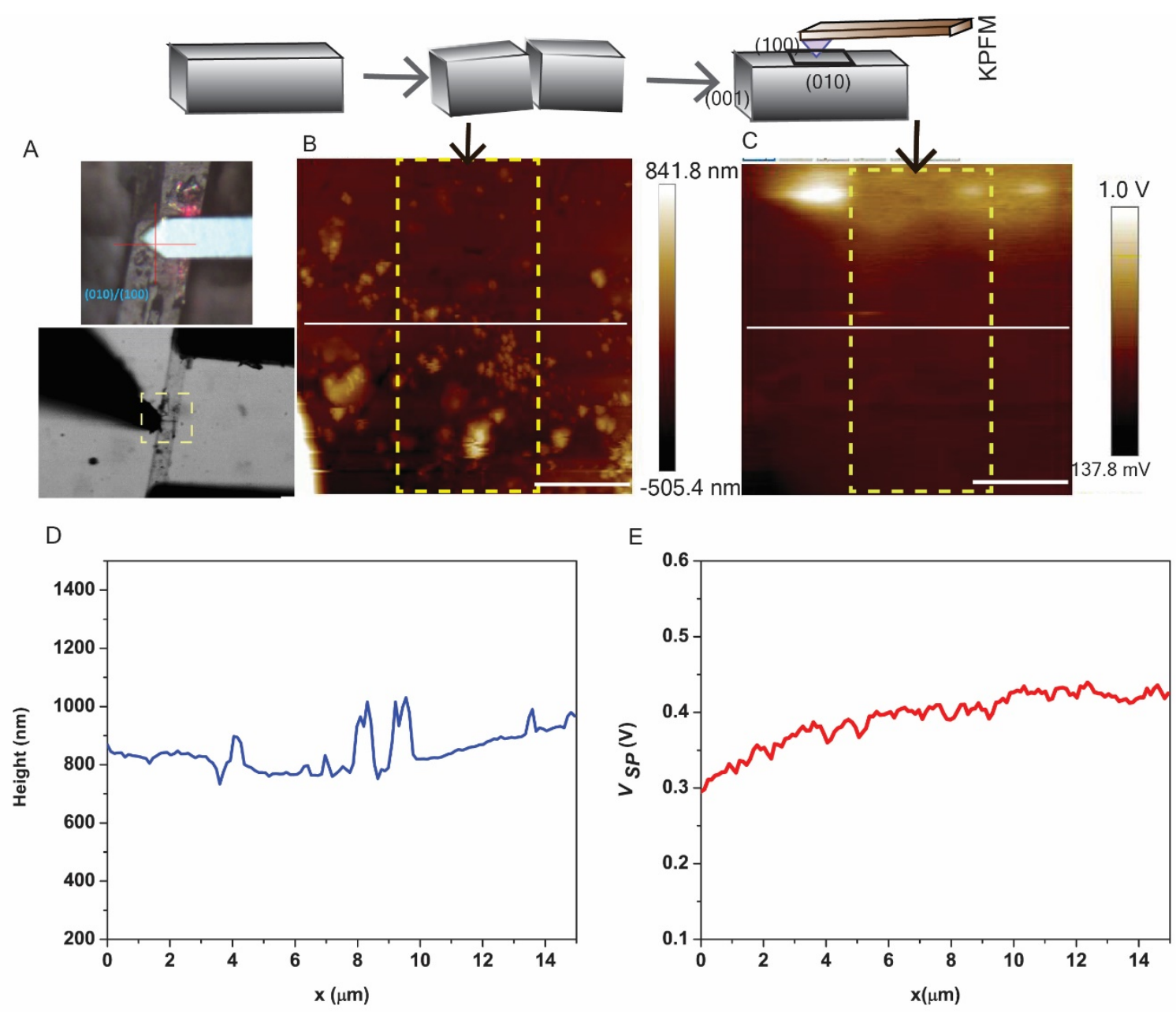

Fig. S11. KPFM on crack line of neatly-healed crystal of 1. A) Optical image (upper panel) of the perfectly healed crystal during experiment and (lower panel) the video grab showing the crack generation before the healing event of the same crystal; (B) AFM surface topography; (C) KPFM surface potential; (D) profiles of height and (E) surface potential across the crack line. Scale bar for $C$ and $D$ is $5 \mu \mathrm{m}$. 

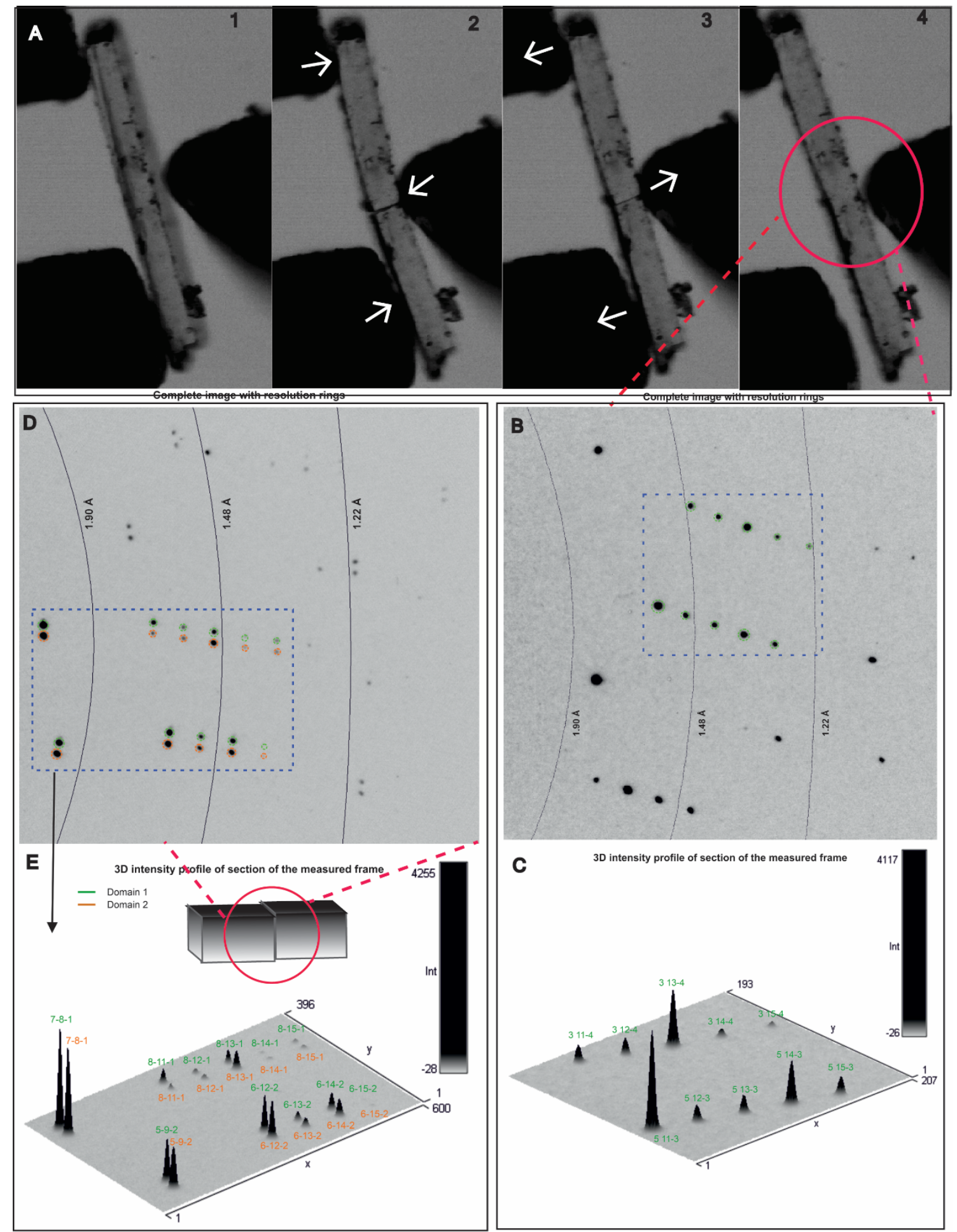

Fig. S12. Healing efficiency evaluation by SCXRD of neatly-healed and partially healed crystals: A) Video grabs (1-4) of the healing event of the crystal used for SCXRD. Image showing the measured 2D diffraction pattern (B) and its corresponding 3D intensity profile $(\mathrm{C})$ for a neatly-healed crystal; Images showing the 2D diffraction pattern (D) and its corresponding 3D intensity profile (E) for an imperfectly-healed crystal with a visible crack line. Corresponding hkl are also indexed. 


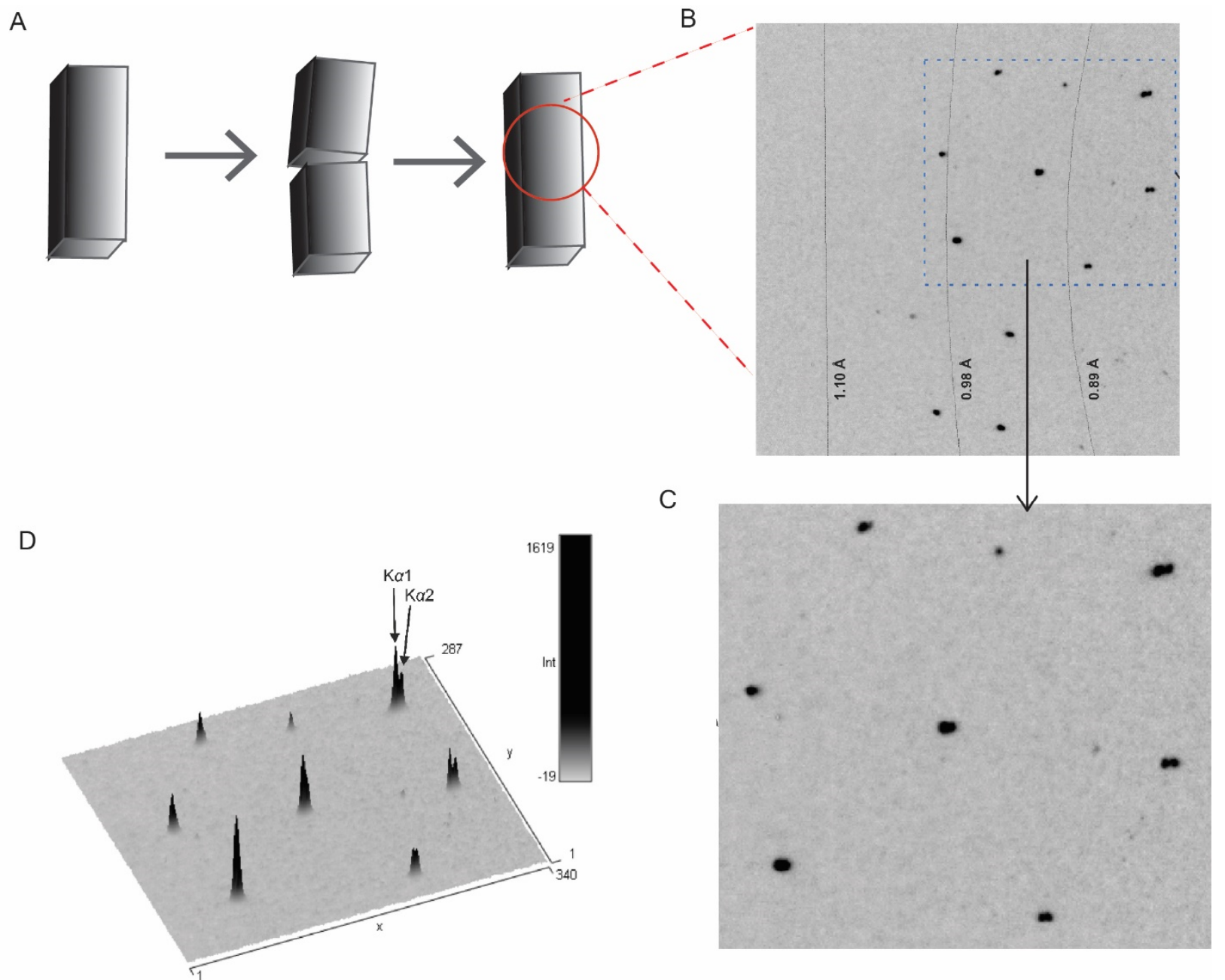

Fig. S13. A) Schematic of self-healing. (B, C) Image showing the measured 2D diffraction pattern $(B)$ and its corresponding $3 D$ intensity profile $(D)$ for a neatlyhealed crystal showing the $2 \mathrm{D}$ diffraction pattern where $K \alpha_{1}$ and $K \alpha_{2}$ were resolved. 


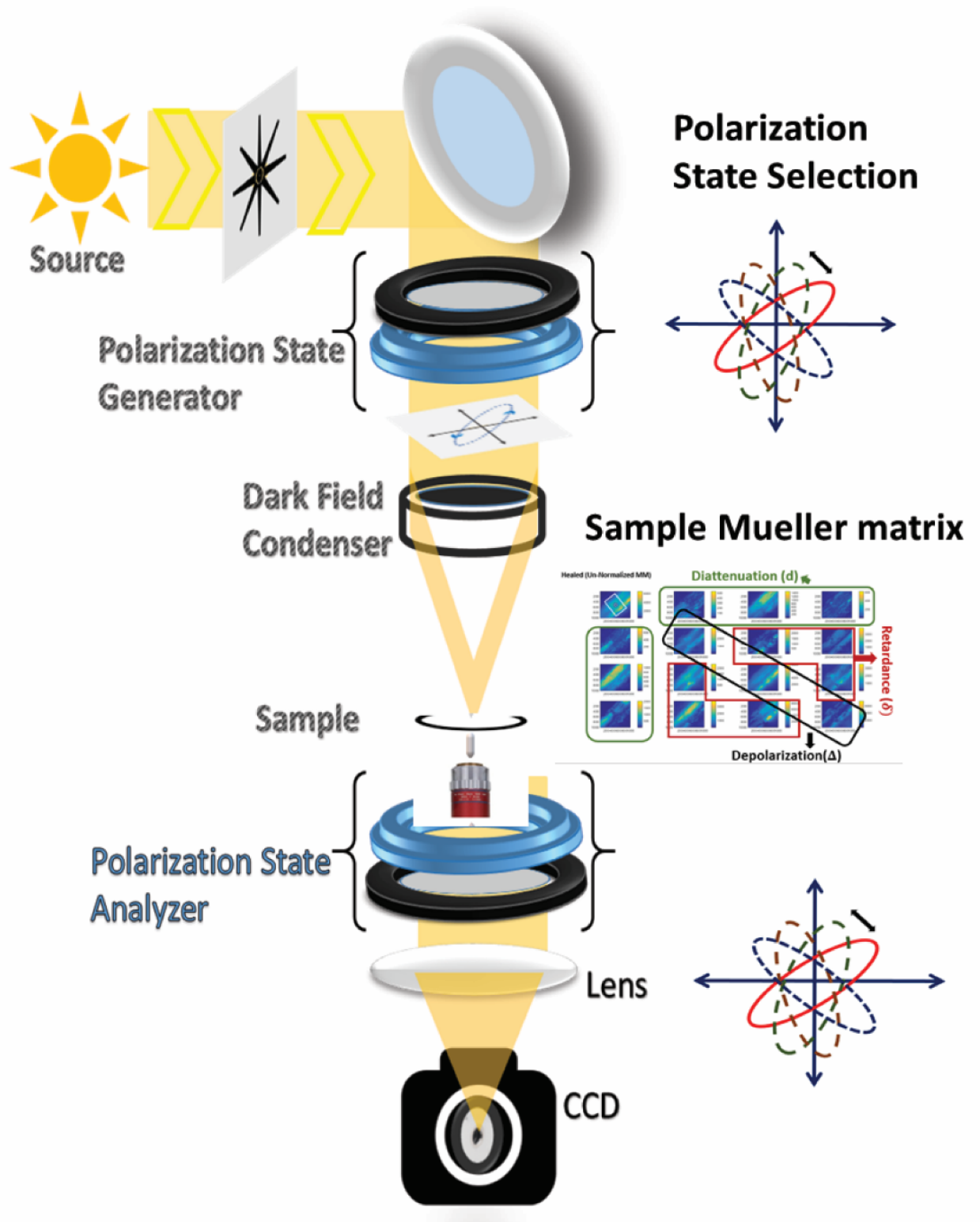

Fig. S14. Schematic representation of Mueller Matrix (MM) measurement instrument setup. 


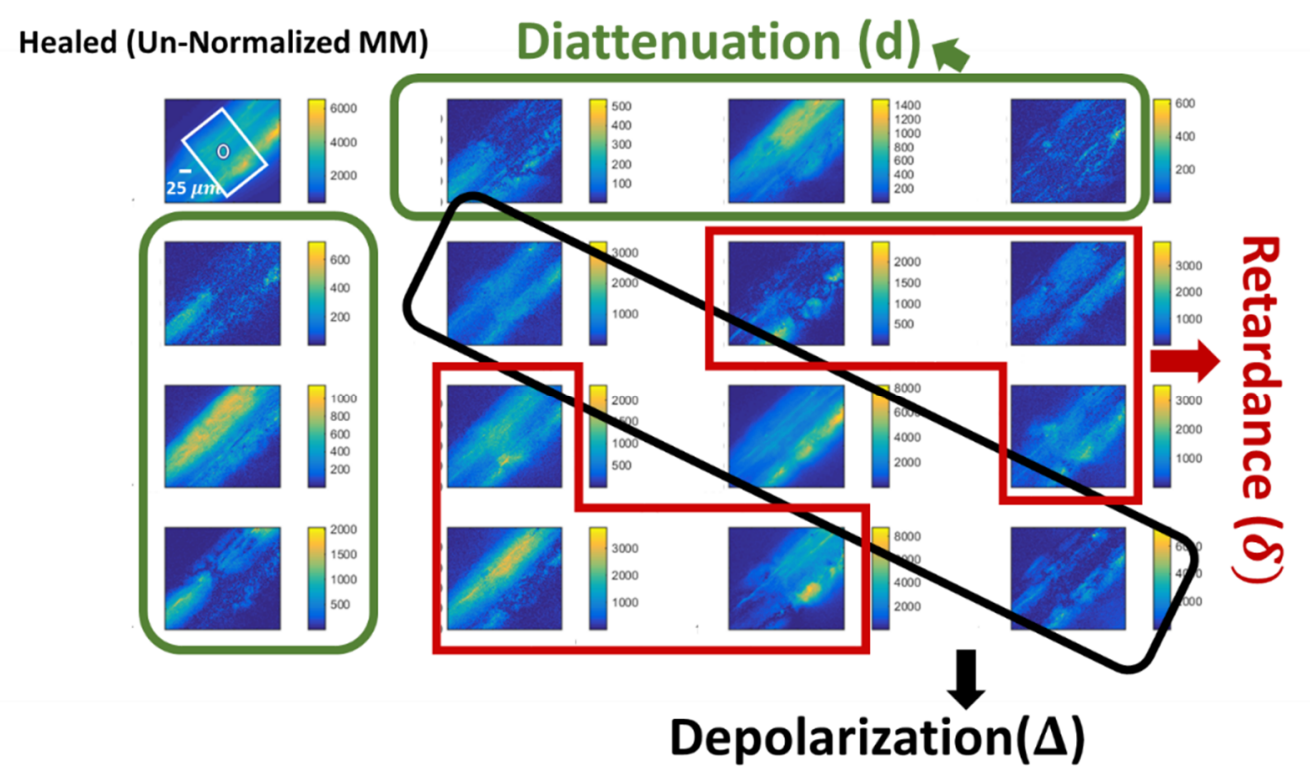

Fig. S15. Complete MM of a healed crystal of $\mathbf{1}$. 


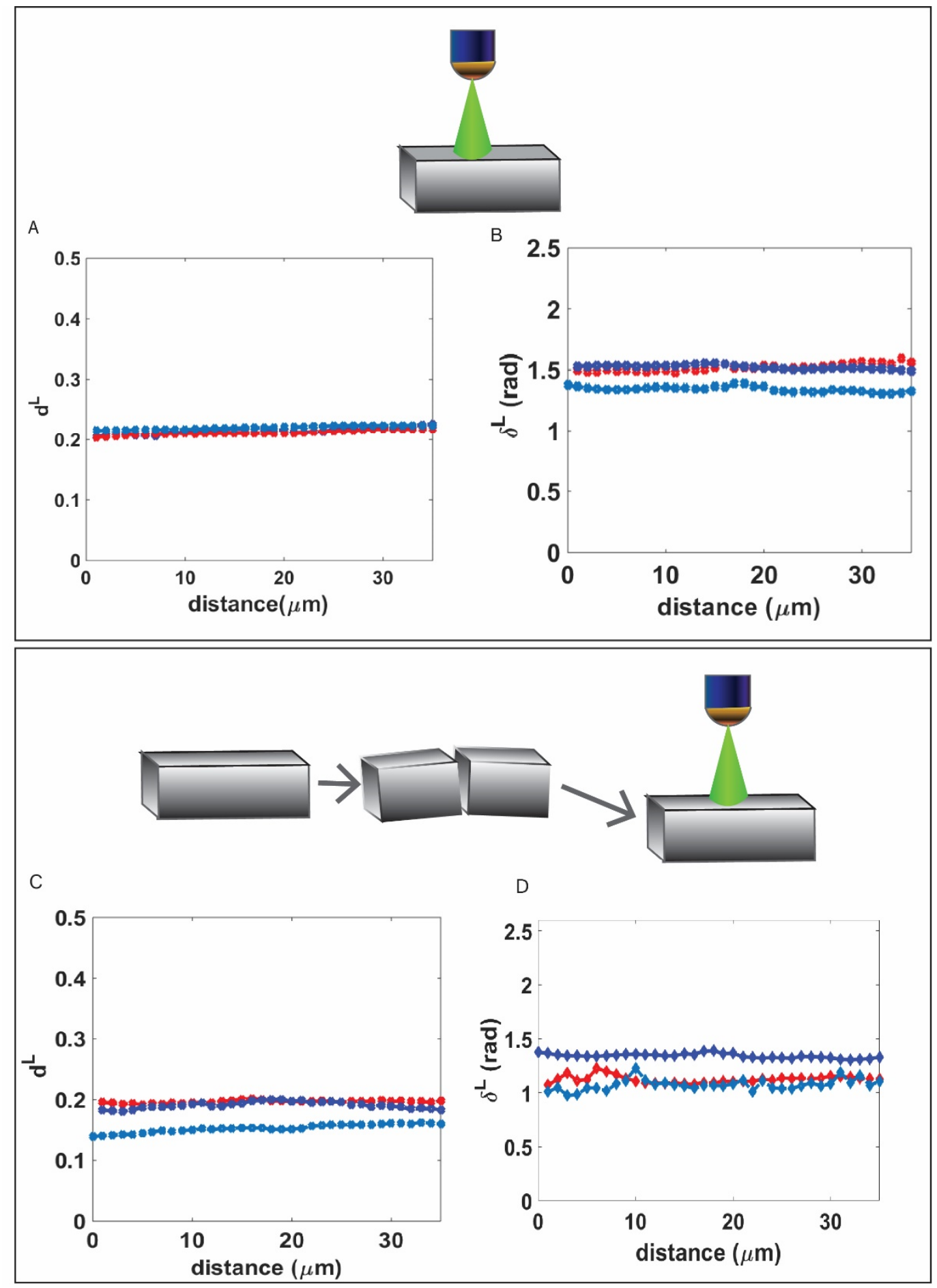

Fig. S16. Plot for spatial map of $d^{L}$ (left panel) and $\delta^{L}$ (right panel) for pristine (A-B) and neatly-healed crystals of three different samples of 1 (C-D). 


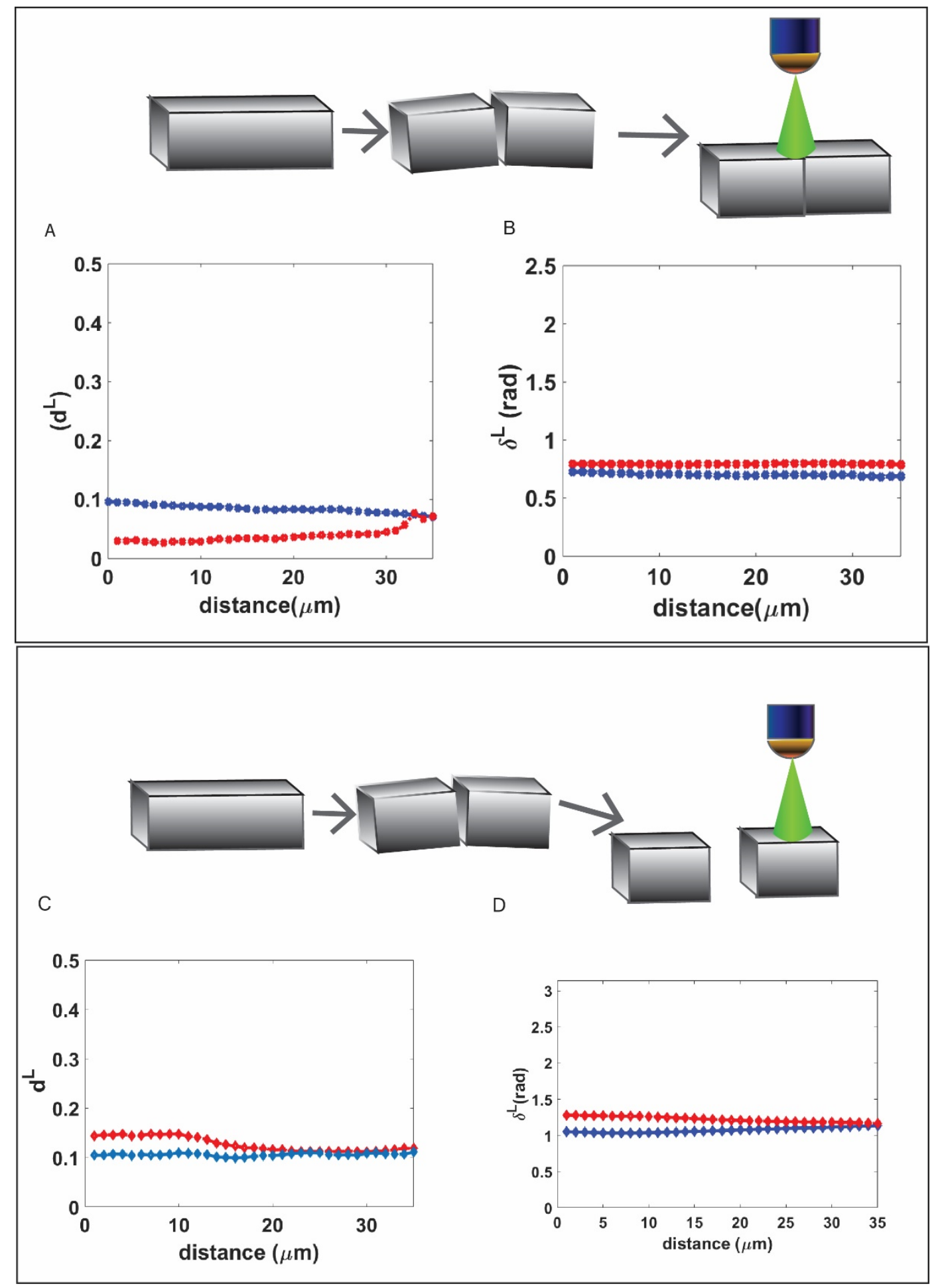

Fig. S17. Plot for spatial map of $d^{L}$ (left panel) and $\delta^{L}$ (right panel) for imperfectly healed crystals $(A-B)$ and cracked and physically separated crystals of two different samples of 1 (C-D). 

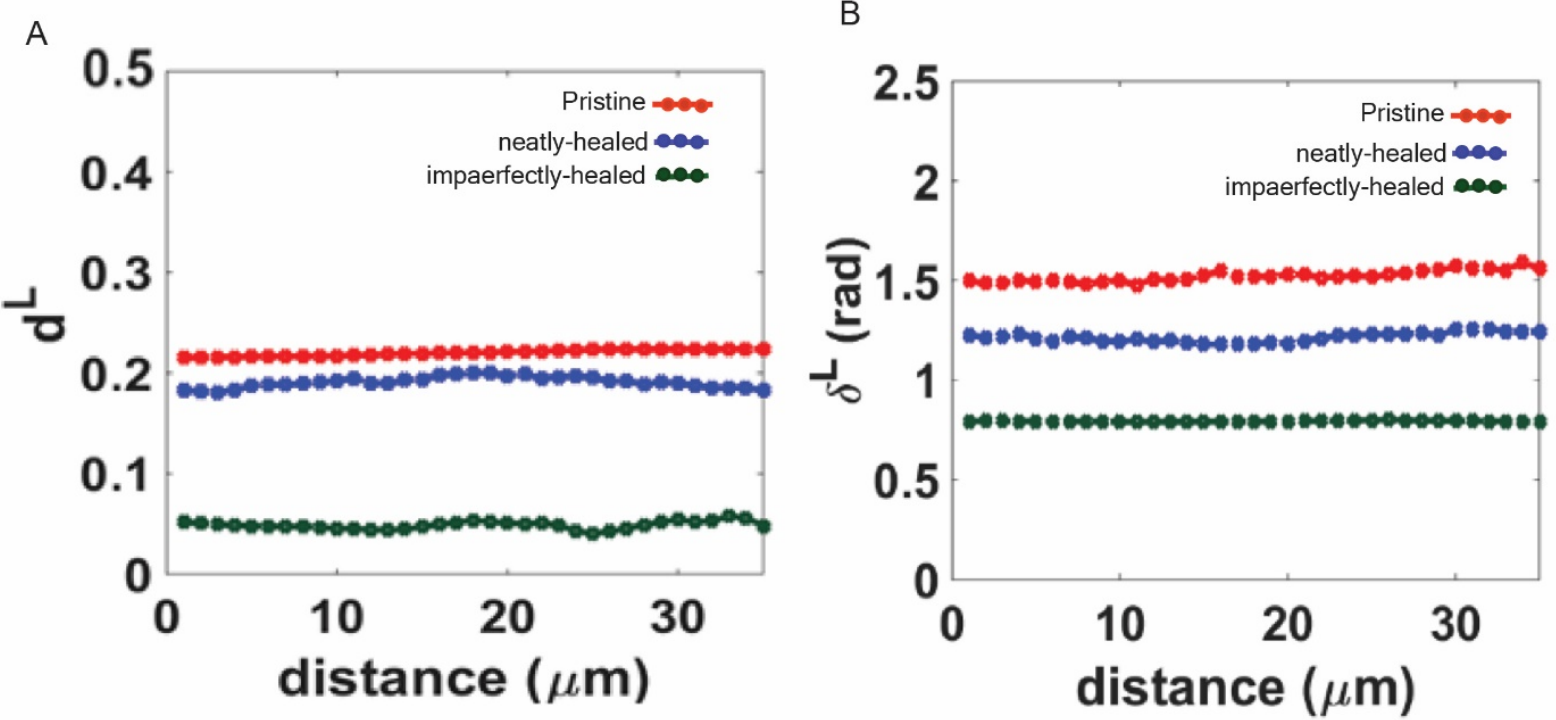

Fig. S18. Comparative plot for spatial map of $d^{L}$ (left panel) (A) and $\delta^{L}$ (right panel) (B) for pristine (red), neatly-healed (blue), imperfectly-healed (dark green) crystals of 1. 


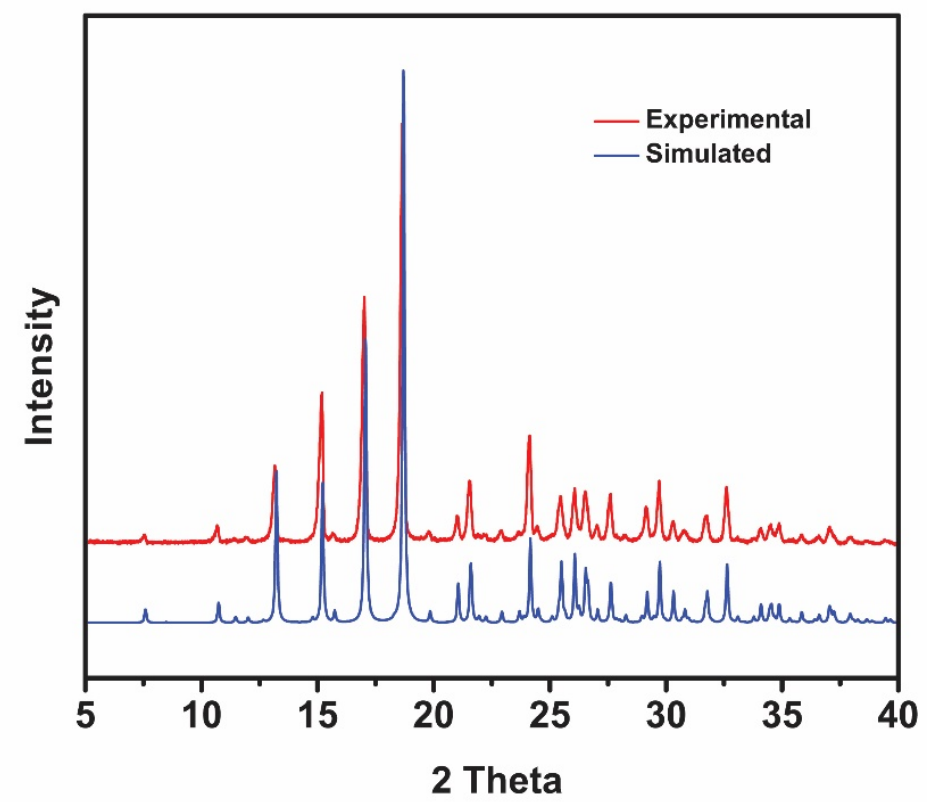

Fig. S19. PXRD pattern of the simulated and experimental data confirming the phase purity of the bulk sample of 1 .

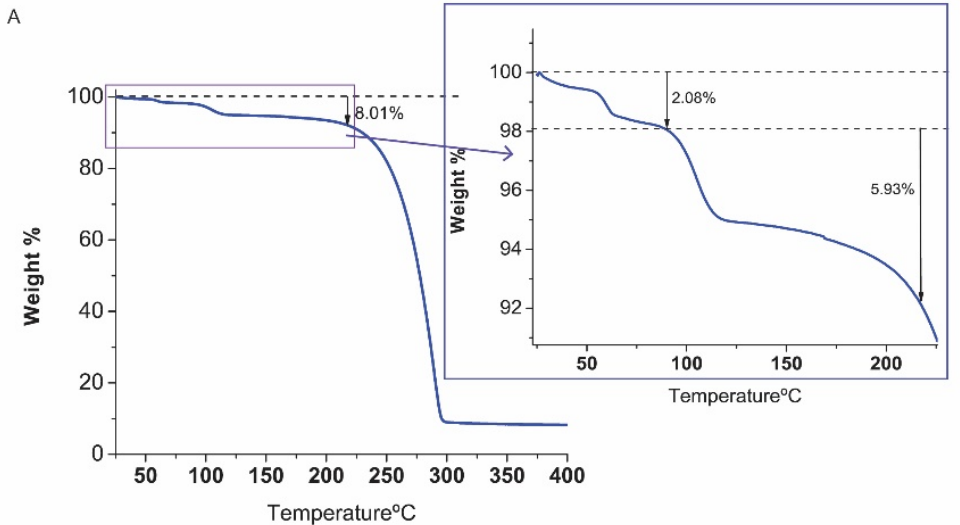

B

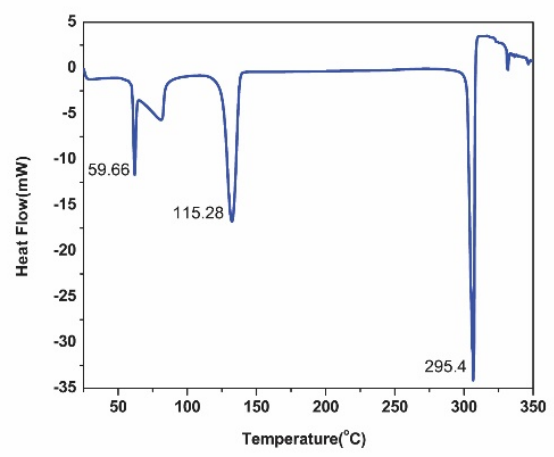

Fig. S20. A) TGA plot of 1 showing the water loss from lattice in steps $\left(<220^{\circ} \mathrm{C}\right)$ well before the melting $\left(\sim 295^{\circ} \mathrm{C}\right.$; onset value) temperature and $\left.\mathrm{B}\right)$ the corresponding endotherms in DSC. 
Table S1. Comparison of hydrogen bond geometries in pristine vs neatly-healed crystal.

\begin{tabular}{|c|c|c|c|c|c|c|c|c|}
\hline \multirow[t]{2}{*}{ D-H $\cdots A$} & \multicolumn{2}{|c|}{$\mathrm{d}(\mathrm{D}-\mathrm{H}) / \mathrm{A}$} & \multicolumn{2}{|c|}{$\mathrm{d}(\mathrm{H} \cdots \mathrm{A}) / \check{A}$} & \multicolumn{2}{|c|}{$\mathrm{d}(\mathrm{D}-\mathrm{A}) / \check{\mathrm{A}}$} & \multicolumn{2}{|c|}{$\mathrm{D}-\mathrm{H} \cdots \mathrm{A} /{ }^{\circ}$} \\
\hline & Pristine & $\begin{array}{l}\text { Neatly- } \\
\text { healed }\end{array}$ & Pristine & $\begin{array}{l}\text { Neatly- } \\
\text { healed }\end{array}$ & Pristine & $\begin{array}{l}\text { Neatly } \\
\text { healed }\end{array}$ & Pristine & $\begin{array}{l}\text { Neatly- } \\
\text { healed }\end{array}$ \\
\hline O1-H1D...N2 & $0.88(4)$ & $1.00(4)$ & $1.99(4)$ & $1.86(4)$ & $2.839(3)$ & $2.836(3)$ & $162(3)$ & $165(3)$ \\
\hline O1-H1E...O2 & $0.92(4)$ & $0.89(4)$ & $1.88(4)$ & $1.89(4)$ & $2.790(3)$ & $2.785(3)$ & 173(3) & $179(4)$ \\
\hline O2-H2A $\cdots$ N10 & $0.81(4)$ & $0.73(4)$ & $2.08(4)$ & $2.16(4)$ & $2.879(3)$ & $2.878(3)$ & $171(3)$ & $168(4)$ \\
\hline $\mathrm{O} 2-\mathrm{H} 2 \mathrm{~B} \cdots \mathrm{O}$ & $0.96(3)$ & $0.93(4)$ & $1.91(3)$ & $1.94(4)$ & $2.857(3)$ & $2.858(3)$ & 166(3) & 168(3) \\
\hline O3-H3A…N14 & $0.98(4)$ & $0.95(4)$ & $1.86(4)$ & $1.89(4)$ & $2.808(3)$ & $2.806(3)$ & $162(3)$ & $161(3)$ \\
\hline O3-H3B $\cdots 04$ & $0.78(4)$ & $0.80(4)$ & $1.99(4)$ & $1.97(4)$ & $2.765(3)$ & $2.763(3)$ & $171(4)$ & $170(4)$ \\
\hline O4-H4A $\cdots$ O $^{1}$ & $0.71(4)$ & $0.76(4)$ & $2.19(4)$ & $2.14(4)$ & $2.895(3)$ & $2.894(3)$ & $172(4)$ & $171(4)$ \\
\hline $\mathrm{N} 1-\mathrm{H} 1 \cdots \mathrm{N} 4^{2}$ & 0.94 & 0.86 & 1.96 & 2.03 & $2.883(3)$ & $2.882(3)$ & 169.0 & 169.5 \\
\hline N5-H5 $\cdots \mathrm{O} 2$ & 0.83 & 0.80 & 2.01 & 2.04 & $2.833(3)$ & $2.832(3)$ & 170.2 & 170.4 \\
\hline N13-H13 $\cdots$ N6 & 0.82 & 0.84 & 2.06 & 2.04 & $2.879(3)$ & $2.876(3)$ & 173.4 & 173.1 \\
\hline
\end{tabular}

${ }^{1}+\mathrm{X},+\mathrm{Y}, 1+\mathrm{Z} ;{ }^{2}+\mathrm{Y}, 1-\mathrm{X},-3 / 4+\mathrm{Z}$ 
Table S2. Comparison of mechanical properties (elastic moduli) of different types of self-healing materials.

\begin{tabular}{|l|l|l|l|l|}
\hline Material type & Stimuli & Modulus & $\begin{array}{l}\text { Healing efficiency } \\
\text { (in the context of } \\
\text { mechanical } \\
\text { property) }\end{array}$ & Ref. \\
\hline Polymer & Heat & $3 \mathrm{GPa}$ & $\sim 50 \%$ & 14 \\
\hline Polymer & Not required & $300 \mathrm{MPa}$ & - & 42 \\
\hline Composite & Heat & $24 \mathrm{GPa}$ & $44 \%$ & 43 \\
\hline Hydrogel & - & In MPa scale & - & 44 \\
\hline Single crystal & Moisture & $2 \mathrm{MPa}$ & $67 \%$ & 16 \\
\hline Single crystal & Not required & $\mathbf{8 - 1 5} \mathrm{GPa}$ & $\begin{array}{l}\sim 85 \% \text { (here in the } \\
\text { context of } \\
\text { nanoscale order) }\end{array}$ & Current work \\
\hline & & & & \\
\hline
\end{tabular}


Table S3. Experimental crystallographic data of pristine and neatly-healed crystals of 1.

\begin{tabular}{|c|c|c|}
\hline Sample state & Pristine & Neatly-healed \\
\hline Formula & \multicolumn{2}{|c|}{$\mathrm{C}_{10} \mathrm{H}_{14} \mathrm{~N}_{4} . \mathrm{H}_{2} \mathrm{O}$} \\
\hline $\begin{array}{l}\text { Crystal } \\
\text { System }\end{array}$ & \multicolumn{2}{|c|}{ tetragonal } \\
\hline $\begin{array}{l}\text { Space } \\
\text { group }\end{array}$ & \multicolumn{2}{|c|}{$P 4_{3}$} \\
\hline$a[\AA$ & 23.09090(10) & $\begin{array}{c}23.08310(10 \\
)\end{array}$ \\
\hline$c[\ddot{A}]$ & $8.62460(10)$ & $8.62120(10)$ \\
\hline$V\left[\check{A}^{3}\right]$ & $4598.55(6)$ & 4593.63(7) \\
\hline$Z$ & 16 & 16 \\
\hline Radiation & \multicolumn{2}{|c|}{ CuKa } \\
\hline $\begin{array}{l}\text { pcalco } \\
{\left[\mathrm{gcm}^{-3}\right]}\end{array}$ & 1.203 & 1.205 \\
\hline$F[000]$ & 1792.0 & 1792.0 \\
\hline$\mu\left[\mathrm{mm}^{-1}\right]$ & 0.663 & 0.664 \\
\hline $2 \theta\left[^{\circ}\right]$ & $\begin{array}{l}5.412 \text { to } \\
132.606\end{array}$ & 5.414 to 131.496 \\
\hline$h k l$ range & $\begin{array}{c}-27 \leq \mathrm{h} \leq 24 \\
-27 \leq \mathrm{k} \leq 26 \\
-10 \leq \mathrm{I} \leq 10\end{array}$ & $\begin{array}{c}-27 \leq \mathrm{h} \leq 27 \\
-27 \leq \mathrm{k} \leq 27 \\
-10 \leq \mathrm{I} \leq 10\end{array}$ \\
\hline$T[\mathrm{~K}]$ & $100.00(10)$ & $100.00(10)$ \\
\hline refins total & 55584 & 53207 \\
\hline $\begin{array}{c}\text { Criterion of } \\
\text { observabili } \\
\text { ty }\end{array}$ & & \\
\hline $\begin{array}{l}\text { Unique } \\
\text { (obs/all) }\end{array}$ & 7952/7986 & 7882/7899 \\
\hline $\begin{array}{c}R_{1} \text { (obs) / } \\
w R_{2} \text { (all) }\end{array}$ & $0.0258 / 0.0679$ & $0.0267 / 0.07022$ \\
\hline GOF & 1.045 & 1.037 \\
\hline
\end{tabular}


CCDC

2044977

2044978

number 
Table S4. Comparison of covalent bond distances in pristine vs neatly-healed crystal

\begin{tabular}{|l|l|l|l|l|l|}
\hline \multicolumn{1}{|c|}{$\begin{array}{c}\text { atom } \\
\text { groups }\end{array}$} & \multicolumn{2}{|c|}{ distance/A } & \multicolumn{2}{c|}{$\begin{array}{c}\text { atom } \\
\text { groups }\end{array}$} & \multicolumn{2}{c|}{ distance/A } \\
\hline N1-N2 & Pristine & $\begin{array}{c}\text { Neatly- } \\
\text { healed }\end{array}$ & & Pristine & $\begin{array}{c}\text { Neatly- } \\
\text { healed }\end{array}$ \\
\hline N1-C2 & $1.367(3)$ & $1.366(3)$ & C13-C18 & $1.460(4)$ & $1.461(4)$ \\
\hline N2-C4 & $1.346(3)$ & $1.342(3)$ & C14-C15 & $1.494(4)$ & $1.492(3)$ \\
\hline N3-N4 & $1.337(3)$ & $1.338(3)$ & C16-C17 & $1.498(4)$ & $1.500(4)$ \\
\hline N3-C7 & $1.361(3)$ & $1.354(3)$ & C17-C18 & $1.384(4)$ & $1.387(4)$ \\
\hline N4-C9 & $1.345(3)$ & $1.351(3)$ & C18-C19 & $1.409(4)$ & $1.406(3)$ \\
\hline C1-C2 & $1.328(4)$ & $1.339(3)$ & C19-C20 & $1.494(4)$ & $1.493(3)$ \\
\hline C2-C3 & $1.496(4)$ & $1.496(4)$ & C31-C32 & $1.503(4)$ & $1.500(4)$ \\
\hline C3-C4 & $1.393(4)$ & $1.391(4)$ & C32-C33 & $1.392(4)$ & $1.395(4)$ \\
\hline C3-C8 & $1.409(4)$ & $1.414(3)$ & C33-C34 & $1.406(4)$ & $1.409(3)$ \\
\hline C4-C5 & $1.473(4)$ & $1.470(4)$ & C33-C38 & $1.461(4)$ & $1.462(4)$ \\
\hline C6-C7 & $1.491(4)$ & $1.487(4)$ & C34-C35 & $1.494(4)$ & $1.493(4)$ \\
\hline C7-C8 & $1.493(4)$ & $1.492(4)$ & C36-C37 & $1.490(4)$ & $1.494(4)$ \\
\hline C8-C9 & $1.382(4)$ & $1.387(4)$ & C37-C38 & $1.387(4)$ & $1.386(3)$ \\
\hline C9-C10 & $1.406(4)$ & $1.407(4)$ & C38-C39 & $1.403(4)$ & $1.407(4)$ \\
\hline N5-N6 & $1.498(4)$ & $1.492(4)$ & C39-C40 & $1.492(4)$ & $1.492(4)$ \\
\hline N5-C12 & $1.362(3)$ & $1.360(3)$ & N9-N10 & $1.360(3)$ & $1.358(3)$ \\
\hline N6-C14 & $1.337(3)$ & $1.337(3)$ & N9-C22 & $1.358(4)$ & $1.361(3)$ \\
\hline N7-N8 & $1.334(3)$ & $1.332(3)$ & N10-C24 & $1.334(3)$ & $1.337(3)$ \\
\hline N7-C17 & $1.365(3)$ & $1.365(3)$ & N11-N12 & $1.366(3)$ & $1.360(3)$ \\
\hline N8-C19 & $1.341(4)$ & $1.341(3)$ & N11-C27 & $1.345(3)$ & $1.344(3)$ \\
\hline N13-N14 & $1.328(3)$ & $1.335(3)$ & N12-C29 & $1.340(3)$ & $1.343(3)$ \\
\hline N13-C32 & $1.362(3)$ & $1.365(3)$ & C21-C22 & $1.498(4)$ & $1.497(4)$ \\
\hline N14-C34 & $1.339(4)$ & $1.337(4)$ & C22-C23 & $1.375(4)$ & $1.377(4)$ \\
\hline N15-N16 & $1.338(4)$ & $1.337(4)$ & C23-C24 & $1.414(4)$ & $1.413(3)$ \\
\hline N15-C37 & $1.360(3)$ & $1.357(3)$ & C23-C28 & $1.467(4)$ & $1.472(4)$ \\
\hline N16-C39 & $1.340(3)$ & $1.341(3)$ & C24-C25 & $1.486(4)$ & $1.493(4)$ \\
\hline C11-C12 & $1.344(4)$ & $1.342(3)$ & C26-C27 & $1.491(4)$ & $1.492(4)$ \\
\hline C12-C13 & $1.396(4)$ & $1.493(4)$ & C27-C28 & $1.385(4)$ & $1.383(4)$ \\
\hline C13-C14 & $1.408(4)$ & $1.393(3)$ & C28-C29 & $1.409(4)$ & $1.406(4)$ \\
\hline & & $1.410(3)$ & C29-C30 & $1.496(4)$ & $1.490(3)$ \\
\hline
\end{tabular}

Table S5. Comparison of bond angles in pristine vs neatly-healed crystal.

\begin{tabular}{|l|r|r|l|l|l|}
\hline Atom & \multicolumn{2}{|c|}{ Angle/ } & Atom & \multicolumn{2}{c|}{ Angle/ } \\
\hline C2-N1-N2 & $111.5(2)$ & $111.7(2)$ & C19-C18-C13 & $126.0(2)$ & $125.9(2)$ \\
\hline C4-N2-N1 & $105.5(2)$ & $105.5(2)$ & N8-C19-C18 & $110.8(2)$ & $110.9(2)$ \\
\hline C7-N3-N4 & $111.9(2)$ & $112.4(2)$ & N8-C19-C20 & $122.5(2)$ & $122.4(2)$ \\
\hline C9-N4-N3 & $105.1(2)$ & $105.1(2)$ & C18-C19-C20 & $126.7(2)$ & $126.7(2)$ \\
\hline N1-C2-C1 & $122.7(2)$ & $122.8(2)$ & N13-C32-C31 & $122.8(3)$ & $122.7(2)$ \\
\hline
\end{tabular}




\begin{tabular}{|c|c|c|c|c|c|}
\hline N1-C2-C3 & $107.4(2)$ & $107.5(2)$ & $32-C 33$ & $108.2(2)$ & $108.4(2)$ \\
\hline C3-C2-C1 & 129.9(2) & $129.7(2)$ & C33-C32-C31 & $128.9(3)$ & $128.9(2)$ \\
\hline C2-C3-C4 & 104.7(2) & $104.5(2)$ & C32-C33-C34 & $104.1(2)$ & $103.9(2)$ \\
\hline C2-C3-C8 & $127.1(3)$ & $127.4(2)$ & C32-C33-C38 & $126.6(2)$ & $126.9(2)$ \\
\hline C4-C3-C8 & $128.1(2)$ & $127.9(2)$ & C34-C33-C38 & 129.2(2) & $129.1(2)$ \\
\hline N2-C4-C3 & $111.0(2)$ & $110.8(2)$ & N14-C34-C33 & $110.8(2)$ & $110.9(2)$ \\
\hline N2-C4-C5 & 121.1(2) & $121.3(2)$ & N14-C34-C35 & $120.8(2)$ & 121.2(2) \\
\hline C3-C4-C5 & 127.9(2) & $127.9(2)$ & C33-C34-C35 & 128.4(3) & $127.9(2)$ \\
\hline N3-C7-C6 & $122.0(2)$ & $122.3(2)$ & N15-C37-C36 & $122.0(2)$ & $122.3(2)$ \\
\hline N3-C7-C8 & 107.0(2) & $106.4(2)$ & N15-C37-C38 & $107.4(2)$ & $107.4(2)$ \\
\hline C8-C7-C6 & $131.0(2)$ & $131.2(2)$ & C38-C37-C36 & $130.5(2)$ & $130.3(2)$ \\
\hline C7-C8-C3 & $129.0(2)$ & 128 & C37-C38-C33 & 128.6(2) & $128.7(2)$ \\
\hline C7-C8-C9 & 104.8(2) & 105.2(2) & C37-C38-C39 & 104.4(2) & $104.4(2)$ \\
\hline C9-C8-C3 & 126.2(2) & $126.1(2)$ & C39-C38-C33 & $126.8(2)$ & $126.8(2)$ \\
\hline N4-C9-C8 & $111.3(2)$ & $110.9(2)$ & N16-C39-C38 & $111.4(2)$ & $111.2(2)$ \\
\hline N4-C9-C10 & $121.5(2)$ & $121.4(2)$ & N16-C39-C40 & 121.6(2) & $122.0(2)$ \\
\hline C8-C9-C10 & 127.2(2) & $127.6(2)$ & C38-C39-C40 & $127.0(2)$ & $126.8(2)$ \\
\hline C12-N5-N6 & 2) & 111 & 10 & $111.5(2)$ & $111.4(2)$ \\
\hline C14-N6-N5 & $105.4(2)$ & $105.6(2)$ & -N9 & $105.5(2)$ & $105.7(2)$ \\
\hline C17-N7-N8 & $110.8(2)$ & $111.2(2)$ & C27-N1 & $112.5(2)$ & $112.5(2)$ \\
\hline C19-N8-N7 & $106.0(2)$ & 105.6(2) & C29-N12-N11 & 104.4(2) & 104.6(2) \\
\hline C32-N13-N14 & $110.8(2)$ & $110.8(2)$ & N9-C22- & $122.5(3)$ & $122.9(3)$ \\
\hline C34-N14-N13 & $106.0(2)$ & $106.0(2)$ & N9 & $107.3(2)$ & $107.1(2)$ \\
\hline C37-N15-N16 & 112 & 112 & $\mathrm{C} 2$ & $130.2(3)$ & $129.9(2)$ \\
\hline C39-N16-N15 & $104.6(2)$ & $104.9(2)$ & C22-C23-C24 & $104.9(2)$ & $105.1(2)$ \\
\hline N5-C12-C11 & $122.9(2)$ & $123.0(2)$ & C22-C23-C28 & 128.2(2) & $128.4(2)$ \\
\hline N5-C12-C13 & 107.3(2) & 107.3(2) & C24-C23-C28 & $126.9(2)$ & $126.5(2)$ \\
\hline C13-C12-C11 & $129.8(2)$ & $129.6(2)$ & N10-C24-C23 & $110.9(2)$ & $110.6(2)$ \\
\hline C12-C13-C14 & $104.4(2)$ & $104.4(2)$ & N10-C24-C25 & $122.6(2)$ & $122.6(2)$ \\
\hline C12-C13-C18 & $129.1(2)$ & $129.1(2)$ & C23-C24-C25 & $126.4(2)$ & $126.7(2)$ \\
\hline C14-C13-C18 & 126 & 126 & & $123.1(2)$ & $123.1(2)$ \\
\hline N6-C14-C13 & 111. & 110 & 27-C28 & 106.9(2) & $106.8(2)$ \\
\hline N6-C14-C15 & $121.2(2)$ & $121.4(2)$ & C28-C27-C26 & $130.0(2)$ & $130.1(2)$ \\
\hline C13-C14-C15 & $127.8(2)$ & $127.8(2)$ & C27-C28-C23 & $128.0(2)$ & $127.8(2)$ \\
\hline N7-C17-C16 & $123.0(2)$ & $123.0(2)$ & C27-C28-C29 & $104.8(2)$ & $105.2(2)$ \\
\hline N7-C17-C18 & $107.9(2)$ & $107.8(2)$ & C29-C28-C23 & $127.1(2)$ & $127.0(2)$ \\
\hline C18-C17-C16 & $129.0(3)$ & $129.3(3)$ & N12-C29-C28 & $111.4(2)$ & $110.9(2)$ \\
\hline C17 & & & & $121.6(2)$ & $121.8(2)$ \\
\hline C17-C18-C19 & 104.4(2) & $104.5(2)$ & C28-C29-C30 & 127.0(2) & $127.3(2)$ \\
\hline
\end{tabular}


Table S6. Comparison of $d^{L}$ and $\delta^{L}$ parameters for pristine, neatly-healed, imperfectly-healed and fracture-separated (not allowed to recombine) crystals of 1 .

\begin{tabular}{|c|c|c|c|}
\hline Type of Crystal & $\begin{array}{l}\text { Serial } \\
\text { no }\end{array}$ & $\begin{array}{l}\text { Linear Diattenuation ( } \mathrm{d}^{L} \\
\text { ) }\end{array}$ & $\begin{array}{l}\text { Linear Retardance } \\
\delta^{L}(\text { rad })\end{array}$ \\
\hline \multirow[t]{4}{*}{ Pristine } & 1 & $0.2185( \pm 0.0074)$ & $1.4988( \pm 0.0439)$ \\
\hline & 2 & $0.2138( \pm 0.0041)$ & $1.4252( \pm 0.1277)$ \\
\hline & 3 & $0.2089( \pm 0.0029)$ & $1.5232( \pm 0.1566)$ \\
\hline & 4 & $0.1989( \pm 0.0058)$ & $1.3516( \pm 0.027)$ \\
\hline \multirow[t]{3}{*}{ Perfectly Healed } & 1 & $0.2181( \pm 0.0034)$ & $1.1331( \pm 0.0395)$ \\
\hline & 2 & $0.189( \pm 0.005)$ & $1.339( \pm 0.1)$ \\
\hline & 3 & $0.149( \pm 0.0127)$ & $1.3016( \pm 0.044)$ \\
\hline \multirow{4}{*}{$\begin{array}{l}\text { Fractured and } \\
\text { separated } \\
\text { Imperfectly } \\
\text { Healed }\end{array}$} & 1 & $0.1254( \pm 0.013)$ & $1.3585( \pm 0.1173)$ \\
\hline & 2 & $0.156( \pm 0.052)$ & $1.1920( \pm 0.054)$ \\
\hline & 1 & $0.041( \pm 0.002)$ & $0.834( \pm 0.064)$ \\
\hline & 2 & $0.07 \quad( \pm 0.008)$ & $0.6954( \pm 0.0145)$ \\
\hline
\end{tabular}




\section{References:}

32. P. E. Kruger, B. Moubaraki, G. D. Fallon, K. S. Murray, Tetranuclear copper (II) complexes incorporating short and long metal-metal separations: Synthesis, structure and magnetism. J. Chem. Soc., Dalton Trans. $713-718$ (2000).

33. O. V. Dolomanov, L. J. Bourhis, R. J. Gildea, J. A. K. Howard, H. Puschmann, OLEX2: a complete structure solution, refinement and analysis program. J. Appl. Crystallogr. 42, 339-341 (2009).

34. G. M. Sheldrick, SHELXT-Integrated space-group and crystal-structure determination. Acta Cryst. A71, 3-8 (2015).

35. W. C. Oliver, G. M. Pharr, An improved technique for determining hardness and elastic modulus using load and displacement sensing indentation experiments. J. Mater. Res. 7, 1564-1583 (1992).

36. S. K. Karan, S. Maiti, O. Kwon, S. Paria, A. Maitra, S. K. Si, Y. Kim, J. K. Kim, B. B. Khatua. Nature driven spider silk as high energy conversion efficient bio-piezoelectric nanogenerator, Nano Energy. 49, 655-666 (2018).

37. S. Guerin, J. O'Donnell, E. U. Haq, C. McKeown, C. Silien, F. M. Rhen, T. Soulimane, S. AM. Tofail, D. Thompson. Racemic amino acid piezoelectric transducer. Phys. Rev. Lett. 122, 047701(2019).

38. S. Chandel, A. K. Singh, A. Agrawal, K. A. Aneeth, A. Gupta, A. Venugopal, N. Ghosh, Mueller matrix spectroscopy of fano resonance in plasmonic oligomers. Opt. Commun. 432, 84-90 (2019).

39. S. Kumar, H. Purwar, R. Ossikovski, I. A. Vitkin, N. Ghosh, Comparative study of differential matrix and extended polar decomposition formalisms for polarimetric characterization of complex tissue-like turbid media. J. Biomed. Opt. 17, 105006 (2012).

40. A. K. Singh, S. Chandel, S. K. Ray, P. Mitra, N. Ghosh, "Mueller Matrix Approach for Engineering Asymmetric Fano-resonance Line Shape in Anisotropic Optical System" in Fano Resonances in Optics and Microwaves, E. Kamenetskii, A. Sadreev, A. Microshnichenko, Eds. (Springer, Cham. 2018), 219, 57-83.

41. P. Thomas, S. Chandel, A. Mallick, S. S. Sreejith, N. Ghosh, S. Roy, Studying the Crystallization of Polyoxometalates from Colloidal Softoxometalates. Cryst. Growth Des. 18, 4068-4075 (2018).

42. C. C. Hornat, M. W. Urban, Entropy and interfacial energy driven self-healable polymers. Nat. Commun. 11, 1028(2020). 